\title{
Molecular Dynamics of Apo-Adenylate Kinase: A Distance Replica Exchange Method for the Free Energy of Conformational Fluctuations
}

\author{
Hongfeng Lou and Robert I. Cukier* \\ Department of Chemistry and the Quantitative Biology Modeling Initiative, Michigan State University, \\ East Lansing, Michigan 48824-1322 \\ Received: July 8, 2006; In Final Form: September 8, 2006
}

\begin{abstract}
A large domain motion in adenylate kinase from E. coli (AKE) is studied with molecular dynamics. AKE undergoes a large-scale rearrangement of its lid and AMP-binding domains when the open form closes over its substrates, AMP, and $\mathrm{Mg}^{2+}$-ATP, whereby the AMP-binding and lid domains come closer to the core. The third domain, the core, is relatively stable during this motion. A reaction coordinate that monitors the distance between the AMP-binding and core domains is selected to be able to compare with the results of energy transfer experiments. Sampling along this reaction coordinate is carried out by using a distance replica exchange method (DREM), where systems that differ by a restraint potential enforcing different reaction coordinate values are independently simulated with periodic attempts at exchange of these systems. Several methods are used to study the efficiency and convergence properties of the DREM simulation and compared with an analogous non-DREM simulation. The DREM greatly accelerates the rate and extent of configurational sampling and leads to equilibrium sampling as measured by monitoring collective modes obtained from a principal coordinate analysis. The potential of mean force along the reaction coordinate reveals a rather flat region for distances from the open to a relatively closed AKE conformation. The potential of mean force for smaller distances has a distinct minimum that is quite close to that found in the closed form X-ray structure. In concert with a decrease in the reaction coordinate distance (AMP-binding-to-core distance) the lid-to-core distance of AKE also decreases. Therefore, apo AKE can fluctuate from its open form to conformations that are quite similar to its closed form X-ray structure, even in the absence of its substrates.
\end{abstract}

\section{Introduction}

Adenylate kinase (AK) catalyzes the reversible transformation.

\section{SCHEME 1}

$$
\left[\mathrm{Mg}^{2+}-\mathrm{ATP}+\mathrm{AMP} \Leftrightarrow \mathrm{Mg}^{2+}-\mathrm{ADP}+\mathrm{ADP}\right]
$$

There are three domains in AKs: core, lid, and AMP-binding (Amp-bd). AKs are thought to be in an open form in the absence of substrates and, when $\mathrm{Mg}^{2+}$-ATP and AMP are present, the lid and Amp-bd domains undergo major conformational rearrangements, resulting in the closed form ternary complex. ${ }^{1}$ Extensive kinetic studies of rabbit AK (with a shorter lid domain than in AKE) indicate that it occurs by an isorandom $\mathrm{Bi} \mathrm{Bi}$ mechanism, in which the two substrates can bind in random order to one isoform while the two products bind in random order to another isoform of the enzyme. ${ }^{2}$ These kinetics suggest that apo-AKE can exist in at least two conformations, with one form binding $\mathrm{Mg}^{2+}$-ATP and AMP and the other $\mathrm{Mg}^{2+}-\mathrm{ADP}$ and ADP. ${ }^{2}$ Other experiments ${ }^{3,4}$ suggest that each of these subensembles encompasses of a variety of conformers. NMR studies also support the existence of a conformational ensemble for the apo enzyme. ${ }^{5}$ The importance of domain conformational changes has been emphasized by recent experiments showing that they, versus the chemical, phosphoryl transfer step, are rate limiting in AKE and can rationalize differences in activity

* Corresponding author. E-mail: cukier@cem.msu.edu. Telephone: 517 355-9715, extension 263. Fax: 517-353-1793. between mesophiles and thermophiles. ${ }^{6}$ Crystallography ${ }^{1}$ and time-resolved dynamic nonradiative excitation energy transfer experiments ${ }^{7}$ show that binding AMP is associated with an initial conformational change. Binding of the next substrate, usually modeled with a binary substrate, mimics $\mathrm{AP}_{5} \mathrm{~A}$ (ATP and AMP linked by a fifth phosphate group), resulting in the formation of the closed, catalytically competent form. The transition from apo (open) to closed forms involves rearrangements with significant lid and Amp-bd domain motion to close the interdomain cleft. ${ }^{8}$ The energy transfer studies of domain closure in AKE were carried out by labeling appropriate pairs of residues, Ala55, a residue in the Amp-bd domain and Val169, which is just below the lid, and distance distributions that reflect the conformational space exploration were obtained. The apoenzyme has a broad distance distribution, with a mean and width around $30 \AA$. The width of this distribution indicates that apoAKE samples a very large conformational ensemble in solution.

Two extremes of a ligand/substrate binding mechanism can be envisaged. The ligand could bind to the apo form and induce the required conformational changes ("induced fit") or it could select from preexisting protein conformations that are already "predisposed" to the ligand ("conformational selection"). Koshland ${ }^{9}$ refined Fisher's lock and key hypothesis into the concept of induced fit, which he defined as a close fit between the protein and ligand that occurs only after conformational changes are induced by the ligand. ${ }^{9}$ On the other hand, it may be that proteins were designed to sample conformations that are predisposed to capture substrates, to yield the final substrate-bound structure. ${ }^{10-13}$ There is evidence for this latter view in proteins such as 
staphylococcal nuclease, ${ }^{14,15}$ calbindin, ${ }^{16}$ adenylate kinase ${ }^{3,4}$ and calmodulin, ${ }^{17}$ and cyclophilin A. ${ }^{13}$ The data noted above on large domain motions in AKE provides a good test system to begin an exploration of these two views of ligand binding. But, if protein molecular dynamics (MD) with explicit solvent is run on the current practical time scale of nanoseconds, simulations of the apo form will tend to stay around that minimum, as we have found in a previous AKE study. ${ }^{18}$ The large rearrangements required to go from the apo to more closed forms cannot be accessed on an MD time scale. That has stimulated a number of studies of adenylate kinase based on the elastic network model (ENM), which is a reduced description relative to atomistic MD and MC approaches, ${ }^{19-21}$ Temiz et al. ${ }^{19}$ studied the open and closed forms with the ENM, Miyashita et al. ${ }^{20}$ connect the two forms using a small set of normal modes that are locally adjusted along a path between the two, and Maragakis and Karplus ${ }^{21}$ use a minimum energy path method to span the two extremes.

The generic sampling problem, which is due to the complexity of the protein's energy landscape, with barriers large compared with the thermal energy separating stable states, is a major concern in MD and Monte Carlo (MC) simulations. A number of methods, such as multicanonical ensemble, ${ }^{22,23}$ simulated tempering, ${ }^{24,25}$ and the replica exchange method (REM), ${ }^{26-29}$ were designed to address this issue. The REM was proposed ${ }^{26,30}$ in the context of Ising spin glasses ${ }^{31}$ and introduced to proteinlike systems by Sugita and Okamoto. ${ }^{27}$ The original versions of the REM used temperature (TREM), and more recently, a Hamiltonian REM (HREM) was introduced. ${ }^{32}$ These methods all contain two elements: (1) Multiple copies of configurations are run independently by MD or MC with different temperatures and/or Hamiltonians. (2) Two neighbors in the sense of temperature and/or Hamiltonian are exchanged, according to the Metropolis-Hastings algorithm. ${ }^{33}$ Different from multicanonical ensemble and simulated tempering methods, the REM does not need to perform trial runs to determine a weight (density of states), as is required in the multicanonical ensemble ${ }^{23}$ and simulated tempering methods. ${ }^{24,25}$ In the TREM, the low-indexed system (usually low temperature) borrows fast equilibration properties from high-indexed systems. That provides a doorway for the low-indexed system to overcome energy barriers and thus improve the sampling. ${ }^{34}$ However, the number of replicas needed in the TREM is unfortunately proportional to the square root of the number of degrees of freedom of the system of interest. ${ }^{32}$

Fukunishi et al..$^{32}$ proposed the HREM, in which the potential function in different Hamiltonians differs by a small subset of the total number of degrees of freedom required to characterize the system. By doing this, one hopes that the number of replicas needed will be reduced. One way to change the Hamiltonian that is well suited to a study where a reaction coordinate is introduced is to integrate the umbrella sampling method ${ }^{33}$ with the HREM. Namely, one creates different systems by adding different umbrella window potential functions to the original system that serve to restrain the systems to different values of the reaction coordinate. We will refer to this specialization of HREM as the distance REM (DREM). This idea was proposed by Sugita and co-workers in the context of a multidimensional temperature and distance replica exchange method and applied to the alanine trimer. ${ }^{35}$

In this work, we use the DREM to investigate if apo-AKE can reach conformations that are similar to those found in the substrate bound structures. Harmonic potentials are used as the window potentials along a reaction coordinate, which is the distance between the mass centers of residues Ala 55 and Val
169 , to match the residues used in the energy transfer experiments. $^{7}$ The potential of mean force along this reaction coordinate over a range of approximately $35 \AA$ is obtained with use of the DREM. The large range of the reaction coordinate spans the open and closed X-ray structure values. Because this application of the DREM is designed to probe a very large conformational change in a protein with an explicit solvent simulation, a careful study of the quality of the simulation and its advantages over conventional umbrella sampling (no DREM) is carried out. We find that the DREM does provide enhanced sampling versus no DREM, as measured by improvements in the speed of decay of time correlation functions and the extent of conformational space exploration. The DREM provides a sufficient enhancement in sampling that, over the simulation length, convergence with respect to motions that reflect the large-scale movement of AKE is achieved.

In Section 2 the DREM is introduced and details of its application to the AKE simulation are provided, along with various methods to investigate its convergence and enhanced sampling quality. Section 3 first addresses the validation of the acceptance ratio in the DREM exchanges and then studies the convergence to equilibrium of the sampling and measures of the DREM sampling improvement versus no-DREM. The potential of mean force is then presented along with related quantities that provide a picture of the motion of AKE in its configuration space. Our conclusions are presented in Section 4.

\section{Methodology}

Distance Replica Exchange Method. The temperature REM (TREM) constructs independent copies of a system that differ by their temperature. The REM concept can be generalized to a Hamiltonian REM, ${ }^{32}$ where the systems differ by their Hamiltonian (in practice, in their potential energy function). As a matter of terminology, we shall refer to these different Hamiltonians as systems (versus replicas) because replica connotes a copy of an item. The term replicas will be reserved for the configurations that are present on any particular MD step. It is perhaps useful to take an operational point of view and consider that each processor on a computer is assigned a particular system (Hamiltonian) and different replicas (configurations) can visit that particular processor (system). In our MD program, CUKMODY, however, a given replica is maintained on a particular processor and the systems (the window function parameters) move onto and out of that processor.

If the desire is to enhance the sampling along a chosen reaction coordinate, then systems that differ by a window potential to maintain a desired distance can be introduced to give a distance REM (DREM). Thus, we add a window potential $W_{i}(\mathbf{r})(i=1,2, \ldots, N)$ to the original Hamiltonian $H_{0}(\mathbf{X})$ where $\mathbf{r}=f(\mathbf{X})$ is any reaction coordinate dependent on $\mathbf{X}$, the configuration point, so that $H_{i}(\mathbf{X})=H_{0}(\mathbf{X})+W_{i}(\mathbf{r})$. As in the HREM, ${ }^{32}$ appeal to the detailed balance condition

$$
\alpha\left(\mathbf{X}_{i} \mathbf{X}_{j} \rightarrow \mathbf{X}_{j} \mathbf{X}_{i}\right) P_{i}\left(\mathbf{X}_{i}\right) P_{j}\left(\mathbf{X}_{j}\right)=\alpha\left(\mathbf{X}_{j} \mathbf{X}_{i} \rightarrow \mathbf{X}_{i} \mathbf{X}_{j}\right) P_{i}\left(\mathbf{X}_{j}\right) P_{j}\left(\mathbf{X}_{i}\right)
$$

where $\alpha\left(\mathbf{X}_{i} \mathbf{X}_{j} \rightarrow \mathbf{X}_{j} \mathbf{X}_{i}\right)$ is the acceptance probability that configuration $\mathbf{X}_{i}$ in the $i$ th system exchanges with configuration $\mathbf{X}_{j}$ in the $j$ th system and $P_{i}(\mathbf{X})$ is the Boltzmann distribution at temperature $T=1 / k_{\mathrm{B}} \beta$ for the $i$ th system provides a rule for the exchange between two systems. In our case of a onedimensional distance reaction coordinate, $r$, the $i$ th window function is chosen as a harmonic restraint $W_{i}(r)=k_{i}\left(r-r_{0}^{i}\right)^{2}$ 
with $k_{i}$ and $r_{0}^{i}$ the force constant and equilibrium distance, respectively. The acceptance probability that follows from eq 2.1 and the harmonic window potential is

$$
\alpha\left(\mathbf{X}_{i} \mathbf{X}_{j} \rightarrow \mathbf{X}_{j} \mathbf{X}_{i}\right)=\min \left(1, e^{-\Delta\left(\mathbf{X}_{i} \mathbf{X}_{j} \rightarrow \mathbf{X}_{j} \mathbf{X}_{i}\right)}\right)
$$

where

$$
\Delta\left(\mathbf{X}_{i} \mathbf{X}_{j} \rightarrow \mathbf{X}_{j} \mathbf{X}_{i}\right)=\beta\left[\left(W_{i}\left(r_{j}\right)+W_{j}\left(r_{i}\right)\right)-\left(W_{i}\left(r_{i}\right)+W_{j}\left(r_{j}\right)\right)\right]
$$

In the case of the same force constant for all windows, eq $2.2 b$ simplifies to

$$
\Delta\left(\mathbf{X}_{i} \mathbf{X}_{j} \rightarrow \mathbf{X}_{j} \mathbf{X}_{i}\right)=\beta k\left(r_{i}-r_{j}\right)\left(r_{0}^{j}-r_{0}^{i}\right)
$$

Between the attempted exchange steps, conventional MD simulations are performed for the different systems. In the TREM (DREM), the exchanges may be thought of as configuration or temperature (window) exchanges. From the computational perspective, it is a great advantage of the DREM that only the force constant and equilibrium distance parameters need be exchanged (eq 2.2b) or just the equilibrium distance parameters (eq 2.2c). The exchanges are attempted between neighboring windows because, for the method to be effective, the overlap between the windows' probability distributions needs to be adequate. In contrast with the TREM (unless ensemble averages at higher temperatures than the "normal" one are of interest), the information from all the windows is used directly because it will ultimately provide the potential of mean force along the reaction coordinate. The details of the DREM application to AKE are given below.

Molecular Dynamics Simulations. The CUKMODY protein molecular dynamics code, which uses the GROMOS $96^{36}$ force field, was modified to incorporate the DREM. The systems are run independently on different nodes of a Linux cluster computer and, when exchanges are attempted, information is passed using the message passing interface technique implemented as MPICH.

Four simulations were performed that we shall refer to as DREMA, DREMP, DREMB, and NoDREM. The simulations were run at $303 \mathrm{~K}$ under fixed number, volume, and temperature $(N V T)$ conditions. ${ }^{37}$ In the DREMA and DREMB, different force constants are used, and the window function parameters are collected in Table 1 . The parameters were chosen by first noting that the root-mean-square position fluctuation for a harmonic potential is $\sqrt{k_{\mathrm{B}} T / k_{i}}$ that, in the absence of other forces, sets the width of the reaction coordinate distribution in a window. The DREMP simulation uses 17 systems with reaction coordinate equilibrium distances from 5 to $21 \AA$ with an increment of $1 \AA$ and uniform force constants of $3 \mathrm{kcal} / \mathrm{mol}$. The uniform force constants for the DREMP simulation turned out to not provide accurate results. Thus, on the basis of short trial runs, additional windows with different force constants to improve the acceptance ratios were added, as discussed below. The NoDREM simulation uses the same window potentials as the DREMB, but does not attempt exchanges. It is a conventional window reaction coordinate simulation, and it was performed to compare its efficiency with a DREM simulation. All the simulations were carried out in a box with sides of $69.05 \AA$, having 9471 waters added. For the evaluation of the electrostatic and the attractive part of the Lennard-Jones energies and forces, the PME method was applied with a direct-space cutoff of 9.0 $\AA$, an Ewald coefficient of 0.32 , and a $72 \times 72 \times 72$ reciprocal space grid. Four $\mathrm{Na}$ ions were added to neutralize the system.
TABLE 1: Mapping of Indices $i$ for the Window Functions $W_{i}(r)$ in the DREMA and DREMB to the Equilibrium

\begin{tabular}{|c|c|c|}
\hline index & $d(\AA)^{a}$ & $k\left(\mathrm{kcal} / \mathrm{mol} \cdot \AA^{2}\right)^{b}$ \\
\hline \multicolumn{3}{|c|}{ DREMA } \\
\hline 0 & 5 & 3 \\
\hline 1 & 5.5 & 6 \\
\hline 2 & 6 & 6 \\
\hline 3 & 6.33 & 20 \\
\hline 4 & 6.66 & 20 \\
\hline 5 & 6.9 & 20 \\
\hline 6 & 7 & 3 \\
\hline 7 & 8 & 3 \\
\hline 8 & 9 & 3 \\
\hline 9 & 9.5 & 6 \\
\hline 10 & 10 & 9 \\
\hline 11 & 10.33 & 20 \\
\hline 12 & 10.66 & 15 \\
\hline 13 & 11 & 3 \\
\hline 14 & 12 & 3 \\
\hline 15 & 13 & 3 \\
\hline 16 & 13.5 & 6 \\
\hline 17 & 14 & 6 \\
\hline 18 & 15 & 3 \\
\hline 19 & 16 & 3 \\
\hline 20 & 17 & 3 \\
\hline 21 & 17.5 & 6 \\
\hline 22 & 18 & 3 \\
\hline \multicolumn{3}{|c|}{ DREMB } \\
\hline 0 & 21 & 3 \\
\hline 1 & 22 & 3 \\
\hline 2 & 23 & 3 \\
\hline 3 & 24 & 3 \\
\hline 4 & 25 & 2 \\
\hline 5 & 26 & 2 \\
\hline 6 & 27 & 2 \\
\hline 7 & 28 & 2 \\
\hline 8 & 29 & 2 \\
\hline 9 & 30 & 3 \\
\hline 10 & 31 & 3 \\
\hline 11 & 32 & 3 \\
\hline 12 & 33 & 3 \\
\hline 13 & 34 & 3 \\
\hline 14 & 35 & 3 \\
\hline 15 & 36 & 3 \\
\hline 16 & 37 & 3 \\
\hline 17 & 38 & 3 \\
\hline 18 & 39 & 3 \\
\hline
\end{tabular}
Distances $\boldsymbol{d}=r_{0}^{i}$ and Force Constants $\boldsymbol{k}$ in the DREM Simulations

${ }^{a}$ Equilibrium distance of the harmonic bias window function. ${ }^{b}$ Force constant of the harmonic bias window function.

To prepare the systems, we start with the open (apo) form $\mathrm{X}$-ray structure (PDB 4AKE, chain A) ${ }^{38}$ where the reaction coordinate distance is $28.7 \AA$, and first relax systems with window equilibrium distances, $r_{0}^{i}$, ranging from 24 to $35 \AA$ for 100 ps. Then, we start with the last trajectory snapshot of the system with $r_{0}^{i}$ of $25 \AA$ and relax the systems with $r_{0}^{i}$ ranging from 18 to $24 \AA$ for another $100 \mathrm{ps}$. The same procedure continues for $r_{0}^{i}$ ranging from 10 to $17 \AA$ and from 5 to $9 \AA$. On the larger distance side, we start with the last trajectory snapshot of the system with equilibrium distance of $35 \AA$ and equilibrate the systems with $r_{0}^{i}$ of 36-39 $\AA$ for 100 ps. For the DREMB and NoDREM, we start the simulations after those preparation times and simulate for 9 and 7 ns, respectively. For the DREMP, another $900 \mathrm{ps}$ are devoted to relaxing the systems without exchange and then run for $7 \mathrm{~ns}$ with exchange. Because of the problems of the DREMP that were found, we added more systems to span some regions of the reaction coordinate. Those extra systems are gently brought to the target force constants and $r_{0}^{i}$ values listed in Table 1, DREMA. Starting from last 
trajectory snapshot of the DREMP of systems with equilibrium distance of 13 and $17 \AA$, two systems with $r_{0}^{i}$ values of 13.5 and $17.5 \AA$ are run with force constants of $3 \mathrm{kcal} / \mathrm{mol}$, and then another $600 \mathrm{ps}$ are run with $6 \mathrm{kcal} / \mathrm{mol}$ force constants. Starting with the last trajectory snapshots of the DREMP of systems with $r_{0}^{i}$ of 7 and $10 \AA$, we first run 200 ps with a force constant of $3 \mathrm{kcal} / \mathrm{mol}$ and $r_{0}^{i}$, respectively, of 6.5 and $10.5 \AA$. Then another $400 \mathrm{ps}$ are run with force constants $6 \mathrm{kcal} / \mathrm{mol}$ and, finally, we fork from those two distances into, respectively, 6.33 and $6.66 \AA$, and 10.33 and $10.66 \AA$, and run for another 200 ps with the target force constants in Table 1. After those extra systems were prepared, we start the DREMA from the last trajectory snapshots of the DREMP and the prepared extra systems. The DREMA was run for $9 \mathrm{~ns}$.

For all the trajectory analyses of AKE, the core is defined as residues 1-29, 60-121, and 160-214, leaving the Amp-bd domain as residues $30-59$ and the lid domain as residues $122-$ 159.

Principal Component Analysis. Principal component analysis $^{39-44}$ (PCA) diagonalizes the covariance matrix $\sigma_{i j}=\left\langle\delta \alpha_{i} \delta \alpha_{j}\right\rangle$ of the atom fluctuations $\delta \alpha_{i}=\alpha_{i}-\left\langle\alpha_{i}\right\rangle$ from their trajectoryaveraged $\left\langle\alpha_{i}(t)\right\rangle=\int_{0}^{T} \alpha_{i}(t) \mathrm{d} t / T$ values, where the $\alpha_{i}=\left\{x_{i}, y_{i}, z_{i}\right\}$ denote the Cartesian components of the position of the $i$ th atom. It decomposes the configuration point $\mathbf{X}(t)=\left(x_{1}(t), y_{1}(t), .\right.$. , $\left.z_{N}(t)\right)^{T}$ as

$$
\mathbf{X}(t)=\sum_{i=1}^{3 N}\left[\mathbf{X}(t) \cdot \mathbf{m}_{i}\right] \mathbf{m}_{i}=\sum_{i=1}^{3 N} p_{i}(t) \mathbf{m}_{i}
$$

where the $\mathbf{m}_{i}$ are the (orthonormal) eigenvectors of the covariance matrix, the corresponding eigenvalues are denoted as $\lambda_{i}{ }^{2}$, and the $p_{i}(t)$ are the mode displacements. The eigenvalues are related to the mean-square fluctuation (MSF) of the atoms over the trajectory as MSF $=(1 / N) \sum_{i} \lambda_{i}{ }^{2}$. In the rotated Cartesian coordinate basis defined by the $\mathbf{m}_{i}(i=1,2, \ldots, 3 N)$, the largest eigenvalue captures the largest fraction of the MSF, the second largest the next largest fraction of the MSF, etc. In favorable cases, a small set of modes capture most of the protein's fluctuation. The lower index modes are associated with collective motions of the protein and their convergence to stable values as a function of simulation length provides a severe test of equilibration of a simulation. In this regard, PCA can be used to assess the improvement in simulation efficiency that the DREM provides relative to a no DREM simulation. Several convergence tests have been proposed. ${ }^{45-47}$ Amadei and coworkers ${ }^{45}$ introduced a root-mean-square inner product (RMSIP) measure

$$
\mathrm{RMSIP}=\left[\frac{1}{-} \sum_{k=1}^{n} \sum_{i=1}^{n} \mathbf{m}_{k}(t) \cdot \mathbf{m}_{i}\left(t^{\prime}\right)\right]^{1 / 2}
$$

that evaluates the overlap of a subset of $n$ modes, where the modes are obtained from different time intervals taken from the total trajectory. For example, time intervals $t^{\prime}$ could be taken from the second half of the trajectory, starting from the end, and the other intervals of length $t$, taken from the first half of the trajectory starting from the beginning. Convergence can be assessed in this manner. The PCA is carried out by using ANALYZER, ${ }^{48}$ a program written for the purpose of analyzing trajectory data by a wide variety of methods.

Methods for Analyzing the Acceptance Ratio of a REM. In a REM simulation, or any method that uses a chain of parametrized simulations that must be connected between an initial and final state, there must be sufficient overlap of probability distributions in the neighboring states. In the REM context, one may use the overlap of the energy distributions at different temperatures for the TREM ${ }^{27}$ and, for DREM, the overlap of the reaction coordinate distribution. ${ }^{35}$ A naive estimate of an acceptance criterion is obtained by simply counting the number of successful exchanges. This method will be referred to as the direct method. A formal measure of the TREM overlap is obtained via the average acceptance ratio

$$
\begin{array}{r}
p_{\text {acc }}=\frac{1}{Z\left(\beta_{0}\right) Z\left(\beta_{1}\right)} \int \mathrm{d} \mathbf{X}_{0} \int \mathrm{d} \mathbf{X}_{1} e^{-\beta_{0} V\left(\mathbf{X}_{0}\right)} e^{-\beta_{1} V\left(\mathbf{X}_{0}\right)} \\
\min \left[1, e^{\left(\beta_{0}-\beta_{0}\right)\left(V\left(\mathbf{X}_{0}\right)-V\left(\mathbf{X}_{0}\right)\right)}\right]
\end{array}
$$

as recently discussed. ${ }^{34} P_{i}(\mathbf{X})=e^{-\beta_{i} V(\mathbf{X})} / Z\left(\beta_{i}\right)$ is the normalized canonical ensemble distribution function, and $Z\left(\beta_{i}\right)$ is the corresponding configurational partition function at temperature $T_{i}=/ k_{\mathrm{B}} \beta_{i}$. With the properties of the min function and by introducing the density of states $\Omega(U)$ as a function of potential energy values $U=V(\mathbf{X})$, one obtains the form presented by Kofke: ${ }^{49}$

$$
\begin{aligned}
& p_{\mathrm{acc}}=\frac{2}{Z\left(\beta_{0}\right) Z\left(\beta_{1}\right)} \int_{U_{m}}^{\infty} \mathrm{d} U_{0} \int_{U_{m}}^{U_{0}} \mathrm{~d} U_{1} \Omega\left(U_{0}\right) \\
& e^{-\beta_{0} U 0 \Omega\left(U_{1}\right)} e^{-\beta_{1} U_{1}}=2 \int_{U_{m}}^{\infty} \mathrm{d} U_{0} \int_{U_{m}}^{U_{0}} \mathrm{~d} U_{1} P_{0}\left(U_{0}\right) P_{1}\left(U_{1}\right)
\end{aligned}
$$

where, by assertion, $\beta_{0}>\beta_{1}\left(T_{0}<T_{1}\right)$, and $U_{m}$ is the minimum possible energy for the system. As Kofke points out, the integral in eq 2.6 quantifies the overlap of the energy distributions in the accepted range of exchanges; thus, this overlapping of the energy distributions is an alternative measure of the acceptance ratio. For the DREM with the same force constants, a similar formula can also be obtained and the acceptance ratio will translate to the overlapping of two reaction coordinate distributions. However, such a relation breaks down when the force constants for the harmonic potential along the reaction coordinate are not uniform. Because, for our DREMA simulation, the force constants are not uniform along the reaction coordinate, we here provide a general version of eq 2.5 that can be applied to any replica exchange method:

$$
p_{\text {acc }}=2 \int_{A} \mathrm{~d} \mathbf{X}_{1} \mathrm{~d} \mathbf{X}_{0} P_{0}\left(\mathbf{X}_{0}\right) P_{1}\left(\mathbf{X}_{1}\right)
$$

where $P_{0}$ and $P_{1}$ are again the density functions of configurations $\mathbf{X}_{0}, \mathbf{X}_{1}$, and $A$ is the area where the acceptance probability $\alpha$ for the Monte Carlo move $\left(\mathbf{X}_{0}, \mathbf{X}_{1}\right) \rightarrow\left(\mathbf{X}_{1}, \mathbf{X}_{0}\right)$ is definitely 1.0, which means that the exchange attempt is definitely accepted under this area. This way of analyzing the acceptance ratio will be referred to as the definite exchange method. In Appendix A, we show that eq 2.7 is a direct result of the detailed balance condition governing the Monte Carlo move in the exchange step. By counting the definite exchanges (exchanges with probability 1) and then doubling the counts, one can get another estimation of the acceptance ratio that in TREM measures the overlapping of the energy distribution and in DREM (with the same force constants) measures the overlapping of the reaction coordinate distribution. Note that, for a sufficiently long trajectory, all methods must lead to the same value of the acceptance ratio. A recent article ${ }^{50}$ on evaluating ratios of partition functions using the REM obtains eq 2.7 along with its generalization that we introduce in Appendix A. The variance of the acceptance probability is analyzed in this work, ${ }^{50}$ and they show that the variance of acceptance probability, whose 
average is given by eq 2.5 , must be less than that obtained from the direct method.

PMF Construction Using WHAM and Its Extension. When a window method is used to obtain a potential of mean force (PMF) along a reaction coordinate, the trajectory data obtained from the different windows needs to be combined. The weighted histogram analysis method (WHAM) ${ }^{51,52}$ combines the data from multiple windows of different bias window potential by writing the true, unbiased (by the window potentials) estimated probability density, $\rho^{(\mathrm{u})}(r)$, along the reaction coordinate $r$ as a linear combination of the window biased probability densities $\rho_{\mathrm{w}}^{(\mathrm{b})}(r)$

$$
\rho^{(\mathrm{u})}(r)=\sum_{w=1}^{N} c_{\mathrm{w}} \rho_{\mathrm{w}}^{(\mathrm{b})}(r)
$$

The coefficients $c_{\mathrm{w}}$ in this linear combination are found by minimizing the statistical error of the density estimation along the reaction coordinate. ${ }^{52} \mathrm{We}$ use the WHAM to obtain the $\operatorname{PMF}(r)=-k_{\mathrm{B}} T \ln \rho^{\mathrm{u}}(r)$ along the reaction coordinate. In Appendix B, we derive an extension of WHAM that provides a multidimensional PMF that depends on coordinates other than the reaction coordinate yet, by assumption, does not require window potentials to restrain these coordinates. That is, the assumption is made that the sampling in these nonrestrained coordinates is reasonable. For example, we show that a simulation with windows along the reaction coordinate $r$ that provide the biased densities $\rho_{\mathrm{w}}^{(\mathrm{b})}(r)(w=1, \ldots, N)$ can provide an unbiased estimate of a PMF in coordinates $x$ and $y$ according to $\operatorname{PMF}(x, y)=-k T \ln \left(\rho^{\mathrm{u}}(x, y)\right)$.

\section{Results}

Acceptance Ratio and Validation of the Simulation. An issue of importance to REM methods is the choice and optimization of the acceptance probability of attempted exchanges. There should be an optimal acceptance probability because, for low exchange probability, the rate of movement through configuration space is small, while for high exchange probability, the movement through configuration space is slow. The tendency is to consider a sufficiently high acceptance ratio as an indication of appropriate performance. Predescu and coworkers $^{34}$ recently analyzed the optimization of the TREM acceptance ratio based on the concept of an effective fraction, defined as the fraction of configurations that leave the lowest temperature system and reach the highest temperature system. Their proof relies on sampling from an equilibrium distribution, with the exchanges attempted sufficiently infrequently that the states involved in exchange are decorrelated and equal acceptance probabilities for attempted neighboring exchanges. On the basis of an analysis of a multidimensional oscillator system, they find that $38.74 \%$ is the optimal acceptance ratio, while an exchange ratio ranging from $7-82 \%$ will only at most double the computational effort. However, this effective fraction concept depends on the fact that, in the TREM, the lowest indexed (lowest temperature) system will borrow the fast equilibration property of the highest indexed (highest temperature) system. This property relies on the feature that the average energy sampled scales with temperature and therefore the region of configuration space sampled by the higher index systems is larger, for a given number of steps, than the lower index ones. Thus, the effective fraction is a very important measure for the effectiveness of the exchange in TREM. However, in the DREM, it is not clear whether the lowest indexed system can borrow any such property from the highest indexed system. In the DREM, the highest index system is sampling a different region of configuration space (not a larger region, as in the TREM) than the lowest index system.

However, as we show below, the DREM dramatically reduces the time autocorrelations of the systems, and the accuracy of the estimation of any time average is inversely proportional to the time autocorrelation. ${ }^{33}$ The reduction of time autocorrelation essentially comes from the fact that different independent replicas are visiting the same particular system. (Recall, per the discussion in the Methods Section, that a system corresponds to a particular Hamiltonian (window function)). Thus, a sufficient acceptance ratio is necessary for the appropriate performance of the simulation. But this does not imply that the larger the acceptance ratio the better the simulation because it is possible that a replica just leaving the system can return to the same system in a near future exchange, which definitely will increase the acceptance ratio, but also, unfortunately, increase the time autocorrelation. Another factor related to the time autocorrelation will be how many replicas visit the same system. With more replicas visiting the same system, there will be an enhanced chance to reduce the time autocorrelation. Thus, we first examine the acceptance ratio, then examine the range of the replicas visiting a particular system, and last, examine the time autocorrelation. It is worth pointing out that the autocorrelations discussed here do not provide information on the decay of the unbiased (true) system time correlations. As in any umbrella sampling based method, only equilibrium information, such as a PMF, is available.

Before we performed the DREMA (see the Methods Section and Table 1) with its added window functions, we carried out the DREMP simulation. It turned out that the DREMP failed due to some insufficient acceptance ratios. For the DREMP, we calculated the acceptance ratios for four time intervals: $3-4$, $4-5,5-6$, and $6-7$ ns. The acceptance ratio was obtained by directly counting the number of actual exchanges performed during a simulation. We refer to this approach as the direct method, as noted in the Methods Section. We also calculate the quantity std/average, which is the ratio of the standard deviation of those acceptance ratios to their averages across the four time intervals. Very large std/averages are observed for exchanges between systems with equilibrium distances of 5 and $6 \AA(86 \%), 6$ and $7 \AA(84 \%), 9$ and $10 \AA$ (104\%), and 10 and $11 \AA(60 \%)$. A detailed examination of the acceptance ratios shows that, across those time intervals, they range from tens or twenties of percent to $1 \%$ for systems with the same window potential. Thus, during the time interval with $1 \%$ acceptance ratio, the system does not have a sufficient acceptance ratio. We also evaluate the distribution of the reaction coordinate for each system for each nanosecond interval. The distributions for the systems at equilibrium distances of 6 and $10 \AA$ are very unstable, with an obvious shift (about $1 \AA$ ) of peaks between the first $5 \mathrm{~ns}$ and last $2 \mathrm{~ns}$ for both systems and a change of fluctuation for the fifth ns for the system with an equilibrium window distance of $6 \AA$. This lack of stability makes the simulation unsuitable for the calculation of a PMF along the reaction coordinate. All these measures show that the DREMP is a problematic simulation. Thus, we performed another simulation (DREMA) based on the DREMP by adding more potential windows along the reaction coordinate and strengthening the force constants where the shifts of peaks appear.

One way to estimate the acceptance ratio is to count directly the number of actual exchanges performed during a simulation, the direct method, just used to investigate the DREMP. The definite exchange method (see Methods Section), which is better 
TABLE 2: Acceptance Ratio for the Time Span of the Whole Simulations

\begin{tabular}{|c|c|c|c|}
\hline potential index & acceptance ratio $^{a}$ & acceptance ratio $^{b}$ & std/average \\
\hline \multicolumn{4}{|c|}{ DREMA $^{c}$} \\
\hline $0 \leftrightarrow 1$ & 0.444 & 0.445 & 0.124 \\
\hline $1 \leftrightarrow 2$ & 0.284 & 0.282 & 0.061 \\
\hline $2 \leftrightarrow 3$ & 0.269 & 0.270 & 0.174 \\
\hline $3 \leftrightarrow 4$ & 0.146 & 0.145 & 0.182 \\
\hline $4 \leftrightarrow 5$ & 0.293 & 0.292 & 0.062 \\
\hline $5 \leftrightarrow 6$ & 0.233 & 0.231 & 0.345 \\
\hline $6 \leftrightarrow 7$ & 0.130 & 0.130 & 0.490 \\
\hline $7 \leftrightarrow 8$ & 0.242 & 0.243 & 0.185 \\
\hline $8 \leftrightarrow 9$ & 0.327 & 0.328 & 0.124 \\
\hline $9 \leftrightarrow 10$ & 0.179 & 0.179 & 0.044 \\
\hline $10 \leftrightarrow 11$ & 0.239 & 0.237 & 0.186 \\
\hline $11 \leftrightarrow 12$ & 0.180 & 0.180 & 0.167 \\
\hline $12 \leftrightarrow 13$ & 0.160 & 0.160 & 0.350 \\
\hline $13 \leftrightarrow 14$ & 0.162 & 0.162 & 0.244 \\
\hline $14 \leftrightarrow 15$ & 0.197 & 0.196 & 0.176 \\
\hline $15 \leftrightarrow 16$ & 0.277 & 0.275 & 0.053 \\
\hline $16 \leftrightarrow 17$ & 0.249 & 0.250 & 0.069 \\
\hline $17 \leftrightarrow 18$ & 0.061 & 0.063 & 0.314 \\
\hline $18 \leftrightarrow 19$ & 0.121 & 0.120 & 0.225 \\
\hline $19 \leftrightarrow 20$ & 0.162 & 0.161 & 0.148 \\
\hline $20 \leftrightarrow 21$ & 0.240 & 0.240 & 0.117 \\
\hline $21 \leftrightarrow 22$ & 0.362 & 0.360 & 0.107 \\
\hline \multicolumn{4}{|c|}{$\mathrm{DREMB}^{d}$} \\
\hline $0 \leftrightarrow 1$ & 0.129 & 0.128 & 0.077 \\
\hline $1 \leftrightarrow 2$ & 0.117 & 0.115 & 0.067 \\
\hline $2 \leftrightarrow 3$ & 0.115 & 0.117 & 0.063 \\
\hline $3 \leftrightarrow 4$ & 0.165 & 0.157 & 0.086 \\
\hline $4 \leftrightarrow 5$ & 0.205 & 0.201 & 0.074 \\
\hline $5 \leftrightarrow 6$ & 0.192 & 0.192 & 0.068 \\
\hline $6 \leftrightarrow 7$ & 0.186 & 0.188 & 0.085 \\
\hline $7 \leftrightarrow 8$ & 0.208 & 0.206 & 0.103 \\
\hline $8 \leftrightarrow 9$ & 0.148 & 0.155 & 0.126 \\
\hline $9 \leftrightarrow 10$ & 0.123 & 0.119 & 0.066 \\
\hline $10 \leftrightarrow 11$ & 0.122 & 0.117 & 0.084 \\
\hline $11 \leftrightarrow 12$ & 0.106 & 0.115 & 0.083 \\
\hline $12 \leftrightarrow 13$ & 0.105 & 0.103 & 0.090 \\
\hline $13 \leftrightarrow 14$ & 0.128 & 0.126 & 0.057 \\
\hline $14 \leftrightarrow 15$ & 0.113 & 0.117 & 0.087 \\
\hline $15 \leftrightarrow 16$ & 0.114 & 0.115 & 0.167 \\
\hline $16 \leftrightarrow 17$ & 0.128 & 0.117 & 0.154 \\
\hline $17 \leftrightarrow 18$ & 0.117 & 0.121 & 0.094 \\
\hline
\end{tabular}

${ }^{a}$ From the direct estimation method. ${ }^{b}$ From the definite exchange method. ${ }^{c}$ DREMA exchanges are attempted every 40 fs. ${ }^{d}$ In the first $7 \mathrm{~ns}$ of DREMB exchanges are attempted every $200 \mathrm{fs}$; for the last 2 ns, every $40 \mathrm{fs}$.

grounded in statistical analysis, evaluates the average acceptance ratio $p_{\text {acc }}$, as given in eqs 2.2 and $2.5-2.7$. The average acceptance ratio defined in eq (2.2) is simply the trajectory average of the acceptance probability $\alpha\left(\mathbf{X}_{i} \mathbf{X}_{j} \rightarrow \mathbf{X}_{j} \mathbf{X}_{i}\right)$ for exchanges between systems $i$ and $j$. The general version of $p_{\text {acc }}$ given in eq 2.7 is suited to the DREM method, where the windows have different force constants, as in the DREMA simulation.

We calculate the acceptance ratio during the whole $9 \mathrm{~ns}$ DREMA and DREMB simulations using both methods. In addition, we calculate the std/average ratios. For the DREMA part, the simulation is divided into five time intervals, $0-1$, $1-3,3-5,5-7$, and $7-9 \mathrm{~ns}$, and in the DREMB part, the simulation is divided into six time intervals, $0-1,1-2,2-3$, $3-5,5-7$, and $7-9$ ns. The acceptance ratio data for each interval is used for the purpose of the std/average calculation. Table 2 summarizes the results. For the DREMA, except for the exchange pair 17 and 18, where the direct method acceptance ratio is low at $6 \%$, the acceptance ratio is larger than $10 \%$ for all other exchange pairs. These estimations are confirmed from the estimation by the definite exchange method. However, the acceptance ratios across the different systems are not uniform, which may imply that future tuning could be done. ${ }^{35}$ We also note that there are still large std/average ratios obtained between systems with potential index $5(6.9 \AA)$ and $6(7 \AA), 6$ and 7 (8 $\AA)$, and 12 (10.66 $\AA$ ) and 13 (11 $\AA$ ) with std/average 34\%, 49\%, $35 \%$, respectively. Those three regions point back to the same problematic regions as in the DREMP simulation. However, the variation is significantly reduced compared to the result from the DREMP simulation. Examining the acceptance ratio data in the different time intervals shows a few low ones, namely 4-9\% for exchange pairs with system indices 17 and 18 during the whole simulation and $6 \%$ for exchange pair 6 and 7 during the 5-6 ns time interval, $7 \%$ for exchange pair 12 and 13 during the 5-6 ns time interval, and 7\% for exchange pair 18 and 19 . All the other 102 acceptance ratios for the different systems in the five time intervals are larger than $10 \%$. These results dramatically contrast with the very low acceptance ratio $(\sim 1 \%)$ in the problematic regions in the DREMP simulation.

For the DREMB simulation, all the direct method acceptance ratios are greater than $10 \%$, which is consistent with the estimations obtained from the definite exchange measure. The acceptance ratio is uniform across systems with different window potentials. Also, the std/average ratios are low, indicating that the acceptance ratios are uniform across the whole simulation time for a system with a particular window potential. This uniformity across time and systems implies that the DREMB is a successful DREM simulation, which will be confirmed by other evidence discussed below.

To examine the number of replicas visiting a particular system (with a particular window function), we list in Table 3 the range of replica indices for each system in the DREMA and DREMB simulations. For the DREMA, no system has all the replicas visiting it. There are basically three groups of systems: group A (potential indices $0-6$ ), where systems have low indexed replicas visiting them, group B (potential indices 7-13), where systems have both high- and low-indexed replicas visiting them, and group $C$ (potential indices 14-22), where systems have high-indexed replicas only visiting them. Group A consists of systems with equilibrium distances ranging from 5 to $7 \AA$, and only replicas originating from systems with equilibrium distances less than or equal to $11 \AA$ visit group A. Most of the systems in this group have about $1 / 3$ of the replicas visiting them, except the system with index 6 has $1 / 2$ of the replicas visiting it. Group $\mathrm{C}$ consists of systems with equilibrium distances ranging from 12 to $18 \AA$, and only replicas originating from systems with equilibrium distances larger than or equal to 9.5 $\AA$ visit group C. While most of the systems in this group have $1 / 2$ the replicas visiting them, the last three systems have only $1 / 3$ of the replicas visiting them.

Figure 1 (left panel) displays three typical time trajectories of replica visits for systems with indices 3, 10, and 18. Figure 1 (right panel) displays the complementary information of how selected replicas are visited by different systems for replicas 4, 11, and 21. From the latter plot, one can conclude that, in a specified range, the replica does undergo a random walk in the reaction coordinate for the DREMA simulation.

For the DREMB simulation, there are also no systems where all the replicas visit a particular system, although all the systems have more than half of the replicas visiting them. In contrast with the DREMA simulation, all the systems have replicas with both higher and lower indices visiting them, which results in a random walk of replicas in the reaction coordinate along the whole reaction coordinate chain. In Figure 2 (left panel), we present replica visits for systems indexed as 8 and 18 as typical 
TABLE 3: Summary of Replicas Visiting Systems with a Particular Window Potential

\begin{tabular}{|c|c|c|c|}
\hline potential index & min replica & max replica & no. of replicas visiting \\
\hline \multicolumn{4}{|c|}{ DREMA } \\
\hline 0 & 0 & 8 & 8 \\
\hline 1 & 0 & 8 & 8 \\
\hline 2 & 0 & 8 & 9 \\
\hline 3 & 0 & 8 & 9 \\
\hline 4 & 0 & 8 & 9 \\
\hline 5 & 0 & 8 & 9 \\
\hline 6 & 0 & 13 & 13 \\
\hline 7 & 1 & 19 & 14 \\
\hline 8 & 1 & 19 & 14 \\
\hline 9 & 1 & 19 & 15 \\
\hline 10 & 3 & 19 & 14 \\
\hline 11 & 5 & 19 & 13 \\
\hline 12 & 5 & 19 & 14 \\
\hline 13 & 5 & 19 & 13 \\
\hline 14 & 9 & 22 & 12 \\
\hline 15 & 9 & 22 & 13 \\
\hline 16 & 11 & 22 & 12 \\
\hline 17 & 11 & 22 & 12 \\
\hline 18 & 11 & 22 & 11 \\
\hline 19 & 11 & 22 & 10 \\
\hline 20 & 11 & 22 & 8 \\
\hline 21 & 11 & 22 & 7 \\
\hline 22 & 11 & 22 & 7 \\
\hline \multicolumn{4}{|c|}{ DREMB } \\
\hline 0 & 0 & 15 & 12 \\
\hline 1 & 0 & 15 & 12 \\
\hline 2 & 0 & 16 & 13 \\
\hline 3 & 0 & 16 & 14 \\
\hline 4 & 0 & 16 & 15 \\
\hline 5 & 0 & 16 & 17 \\
\hline 6 & 0 & 17 & 18 \\
\hline 7 & 0 & 17 & 18 \\
\hline 8 & 0 & 17 & 18 \\
\hline 9 & 0 & 17 & 17 \\
\hline 10 & 0 & 18 & 18 \\
\hline 11 & 0 & 18 & 18 \\
\hline 12 & 2 & 18 & 17 \\
\hline 13 & 2 & 18 & 17 \\
\hline 14 & 3 & 18 & 15 \\
\hline 15 & 3 & 18 & 14 \\
\hline 16 & 3 & 18 & 11 \\
\hline 17 & 3 & 18 & 10 \\
\hline 18 & 3 & 18 & 10 \\
\hline
\end{tabular}

examples of the worst and best cases and, in the right panel, systems visiting replicas indexed as 4 and 18 as typical examples of the best and worst cases. One feature of the number of replicas having visited the systems in both the DREMA and DREMB simulations is that systems in the middle of the chain tend to have more replicas visiting them, while systems at the two ends of the chain tend to have fewer replicas visiting them. This feature, as shown below, coincides with the trend of the volume sampled in the space spanned by the first 2 PCA modes.

As noted above, one advantage of the DREM may be to reduce the time autocorrelation of each system. As a test of this idea, we construct (normalized) time autocorrelation functions $(1 / T) \int_{0}^{\mathrm{T}} p_{1}(s) p_{1}(s+t) \mathrm{d} s / \int_{0}^{\mathrm{T}} p_{1}(s) p_{1}(s) \mathrm{d} s$ of the first PCA mode $p_{1}(t)$, defined in eq 2.3. This variable is chosen because the first mode captures the largest fraction of the total MSF of the protein. It also should represent the slowest motions and may be most sensitive to slow decorrelations. Figure 3 displays typical time autocorrelation plots of the displacement of the first mode for a particular system. The trajectory was first fit on the core CA atoms of the open form of AKE before the PCA analysis was performed to better single out the fluctuations of the lid and Amp-bd domains. Figure $3 \mathrm{a}$ is a representative of the five systems, indexed as 5, 6, 9, 10, and 13. Here, one first observes that the time autocorrelation decays toward zero rapidly at the beginning. However, there is a very slow motion taking place, which prevents the correlation from decaying to zero on the simulation time scale. Those five systems coincide with the exchange pairs having large std/average (see Table 2). This is understandable in the sense that a slow motion is taking place in those systems, which makes the equilibration time longer than for other systems. Figure $3 b$ displays a representative of the other 18 systems, which not only have a fast initial decay but also converge to zero very rapidly. For the DREMB simulation, with the exception of system 18, all systems exhibit a fast initial decay and approach zero, as will be discussed more fully when a comparison is made with the NoDREM simulation.

In summary, for the DREMA simulation, significant acceptance ratios are observed between different exchange pairs. A large improvement over the DREMP is gained by adding more systems and adjusting the umbrella sampling force constant and distance parameters. The systems can be divided into 3 groups, two of which have a clear separation in the origin of the replicas visiting them. In contrast to the TREM, though, whether such a separation is an indication of a problem with the DREM is not clear. Whether mixing is significantly helped by a replica going from a low- to a high-indexed system, versus a more limited set of exchanges, is not evident. In terms of the decay of time autocorrelations, all the systems have a large reduction of time autocorrelation relative to non-DREM simulations (see below). For 18 of the 23 systems, the approach to zero also is fast. On the basis of these considerations of acceptance ratio and time autocorrelation, we consider that the DREMA simulation is successful, although there is still room to tune it. For the DREMB, the significant acceptance ratios, their uniformity across time and systems, the successful random walk of the replicas in the reaction coordinate, and the reduction of time autocorrelation definitely indicate that it is a valid simulation.

PCA Comparison of the DREMB and NoDREM Trajectories. To study the ability of the DREM to increase sampling efficiency, we performed a principal component analysis ${ }^{40,42}$ study on every system in both the DREMB and NoDREM (without exchange attempts) simulations. The PCA was performed after the trajectories were fit on the core CA atoms of the open form X-ray structure, which removed the overall rotation and translation and serves to emphasize the motion of the lid and Amp-bd domains. The data from the 1-6 ns intervals of the DREM and NoDREM simulations are used, with the first ns discarded as an equilibration period.

PCA is a powerful tool to study protein dynamics because it can reduce the high-dimensional configuration space to an essential subspace that contains most of the significant, largescale motions. Unfortunately, the slow relaxation of those large fluctuations prevents the fast convergence of the covariance matrix, ${ }^{53}$ which results in slow convergence of the essential space spanned by those large fluctuation modes and may also spoil the detection of fast motions. ${ }^{53}$ Because proteins have a wide distribution of relaxation times, ranging from picoseconds to seconds, ${ }^{54}$ slow relaxation is an intrinsic problem of conventional MD simulations.

In our examination of the efficiency of the DREM, we will take the space spanned by the first two PCA modes as the essential subspace. In Figure 4, the fraction of the total fluctuation, as measured by the MSF, taken up by the first two modes in the DREMB and NoDREM simulations are shown. (The fraction is based on the MSF because this expresses the proportion of the overall fluctuation due to a given number of 


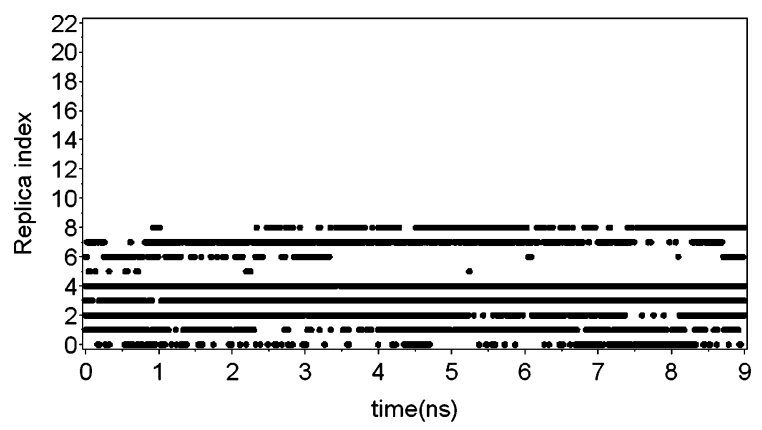

(a)

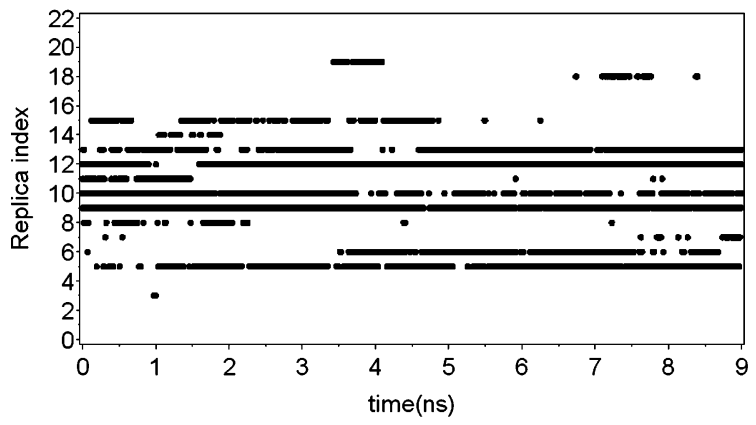

(b)

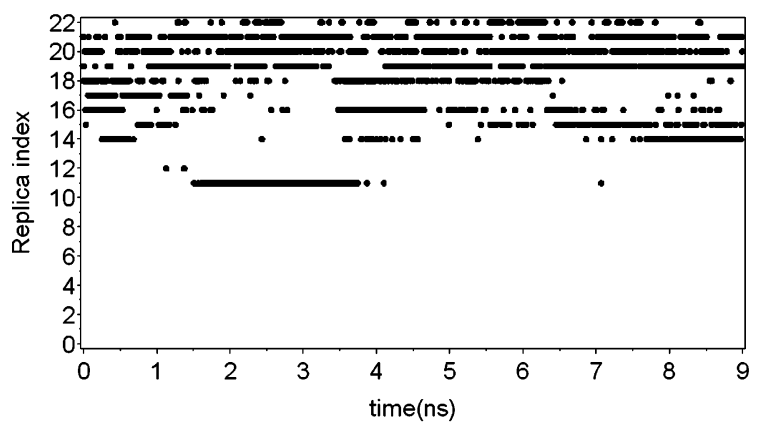

(c)

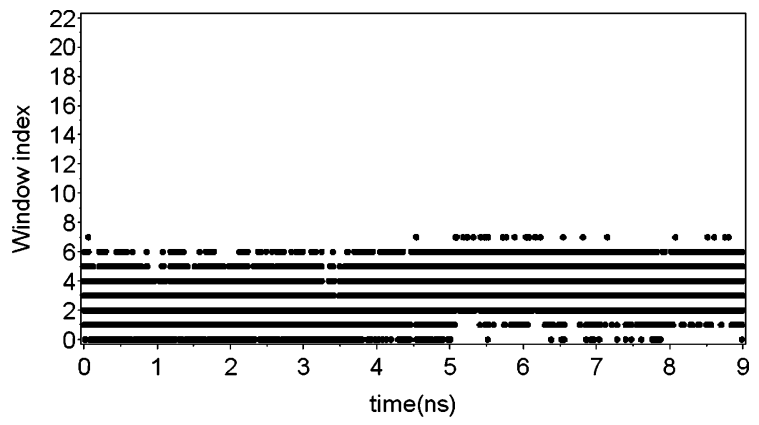

(e)

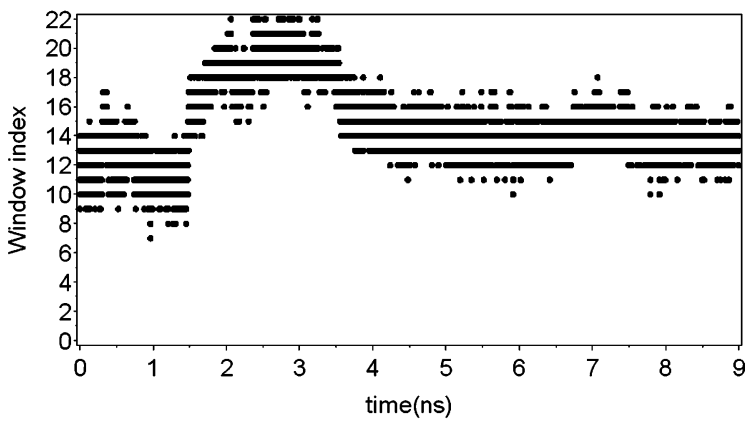

(f)

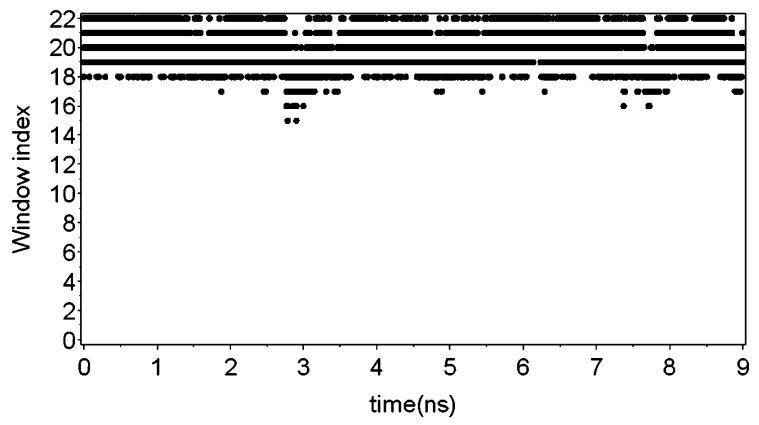

(g)

Figure 1. Visits of the replicas to systems with their window potential indexed as (a) 3, (b) 10, and (c) 18, in the DREMA simulation. Migration patterns of systems for the replicas indexed as (e) 4, (f) 11, (g) 21 , showing the itineration along the reaction coordinate. Note that, in view of the number of data points that are plotted, it may appear that at a particular time several replicas occupy the same window or several systems visit the same replica; this does not happen.

PCA modes.) The smallest proportion in the DREMB is $58 \%$ at the equilibrium window distance of $r_{0}^{i}=37 \AA$, while the smallest in the NoDREM is $49 \%$ at $r_{0}^{i}=31 \AA$. This validates our use of the first two modes as a measure for exploration of the efficiency of the DREM compared with the NoDREM.

Convergence in the essential subspace is a major concern of the application of PCA to protein simulations. The RMSIP measure ${ }^{45}$ discussed in the Methods Section, eq 2.4, examines convergence by comparing the overlap of modes constructed by using different time intervals of the simulation. In our test, $n=2$ (the first two modes) and we take intervals increasing with a $100 \mathrm{ps}$ stride until the intervals are $1 \mathrm{~ns}$ long. After the $1 \mathrm{~ns}$ interval, we take a different stride of $200 \mathrm{ps}$. The two intervals taken to compare with each other are always of same time length, with one starting at the beginning of the analysis time and the other from the end of this analysis time. This arrangement is used to reduce the possible correlation between the two time intervals ${ }^{45}$ intended for comparison. Instead of using the RMSIP, we use its square because RMSIP ${ }^{2}$ is a direct measure of the portion of the projection of the basis of one subspace onto the other subspace. In Figure 5, we show the RMSIP $^{2}$ test for every system in DREMB and NoDREM. In the study of Amadei and co-workers, ${ }^{45}$ they consider that an
RMSIP around $0.6\left(\right.$ RMSIP $\left.^{2}=0.36\right)$ represents good overlap for PCA analysis of convergence. In this sense, all the PCA results, both in DREMB and NoDREM, have good convergence except for the NoDREM at a distance $23 \AA$. For the DREMB, except for the last three systems with equilibrium distance at 37,38 , and $39 \AA$, all the RMSIP ${ }^{2}$ reach about 0.8 . This definitely shows that the DREMB simulation has very good convergence properties in terms of the essential space spanned by the first two PCA modes. We also notice a striking feature of the convergence test that, except for the system at distance $38 \AA$, the DREMB systems have a better or equal convergence level with the corresponding NoDREM systems. Thus, we conclude that, in the DREMB run, the PCA converges better than in the NoDREM. For most of the DREMB data, the plots in Figure 5 appear to be converging toward unity. Because the RMSIP procedure is based on comparing the first half of the data with the second half (here, 2.5 out of the total $5 \mathrm{~ns}$ ), it seems safe to conclude that, over the total $5 \mathrm{~ns}$ interval, the convergence is excellent.

The DREM method should reduce time autocorrelations, implying a speedup in the sampling of configuration space. That is, by moving more rapidly through configuration space, the DREM should decorrelate configurational variables more quickly 


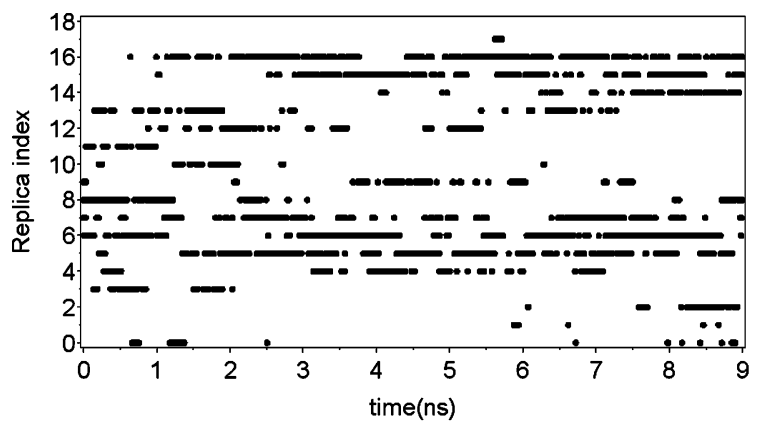

(a)

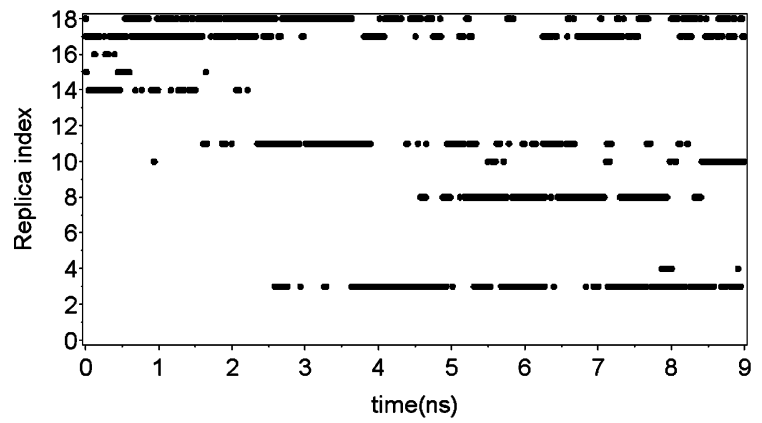

(b)

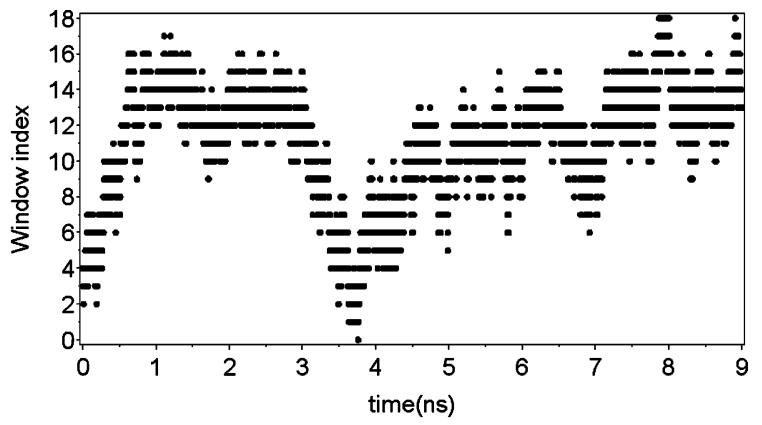

(c)

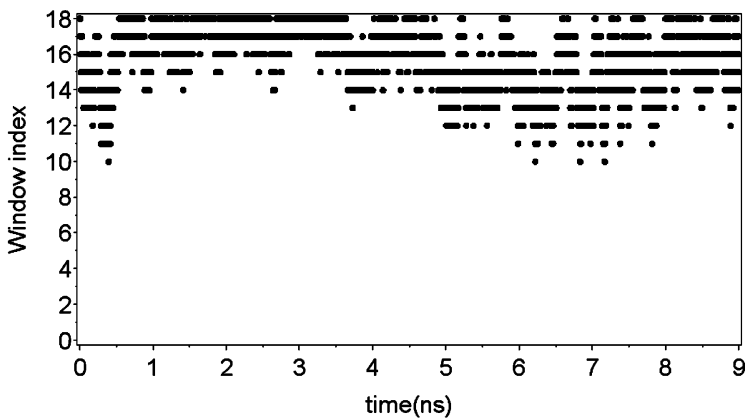

(d)

Figure 2. Best and worst case visits of the replicas with system window potentials indexed as (a) 8 and (b) 18 in the DREMB simulation. Best and worst cases of migration of replicas indexed as (c) 4 and (d) 18. Note that, in view of the number of data points that are plotted, it may appear that at a particular time several replicas occupy the same window or several systems visit the same replica; this does not happen.

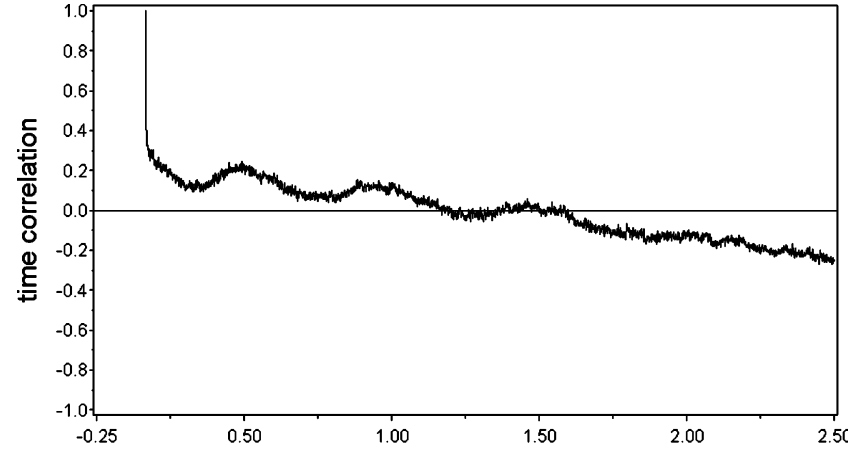

(a)

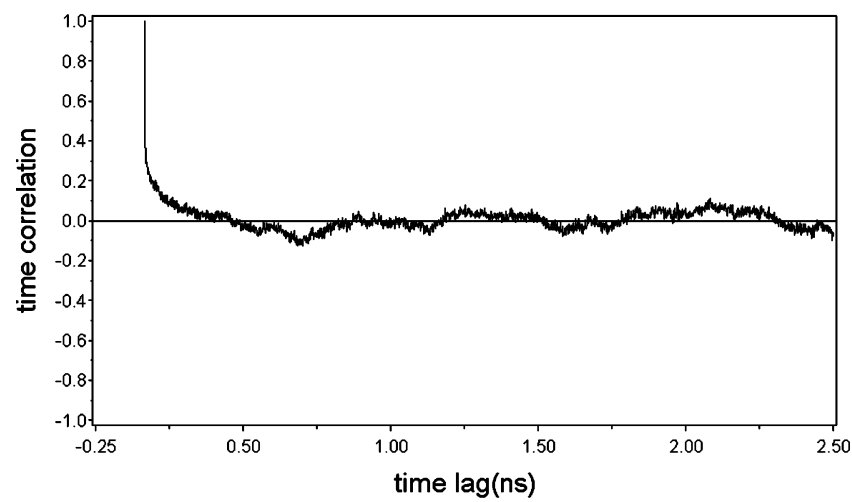

(b)

Figure 3. Time autocorrelation functions of the first PCA mode displacement, $p_{1}(t)$, for the DREMA simulation. (a) A representative of the slow convergence to zero, including systems indexed as 5, 6, 9, 10, and 13. (b) A representative of the fast convergence to zero for all the other 18 systems.

than a normal MD simulation would. By definition, the lower PCA modes describe the largest collective motions of the protein

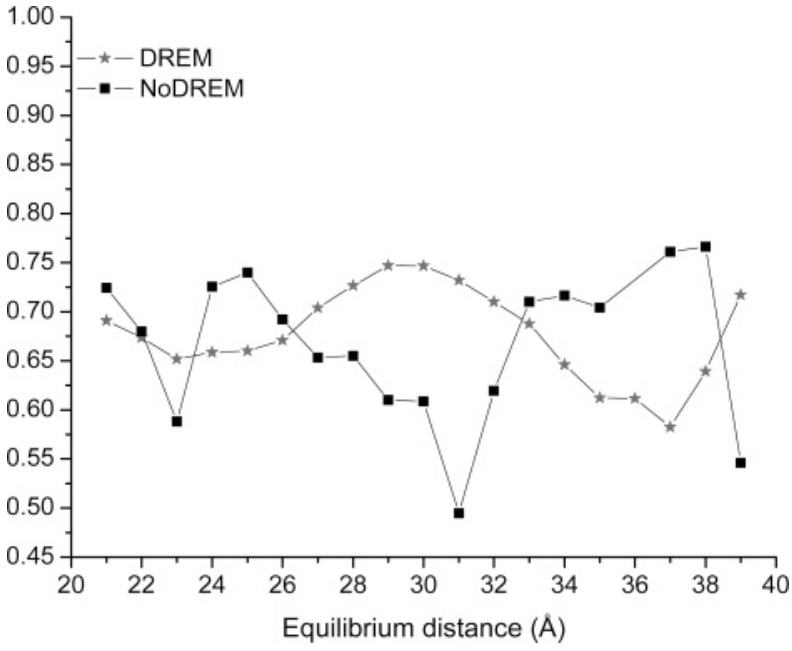

Figure 4. Fraction of the MSF taken up by the first two PCA modes. and should be most subject to the slow convergence problem noted above, and should provide a very sensitive test of the idea of reducing time autocorrelation. Therefore, we focus on the first two PCA modes. In Figure 6, we present representative time autocorrelations of the trajectories for the first two PCA modes for every system in the DREMB and NoDREM simulations. For the first mode (Figure 6a) of the DREMB simulation, with the exceptions for the window equilibrium distances of 34,35 , and $39 \AA$, all the other 16 systems have very rapidly decaying time autocorrelation functions, which correspond to fast relaxation times. On the other hand, all the time autocorrelation functions in the NoDREM simulation decay very slowly. Most of the time, the autocorrelation does not even approach zero during the half of the analysis time window that is used for constructing Figure 6. (We present only half of the analysis time window due to the much greater error in the estimation of time correlation in the latter half of the analysis time window). 

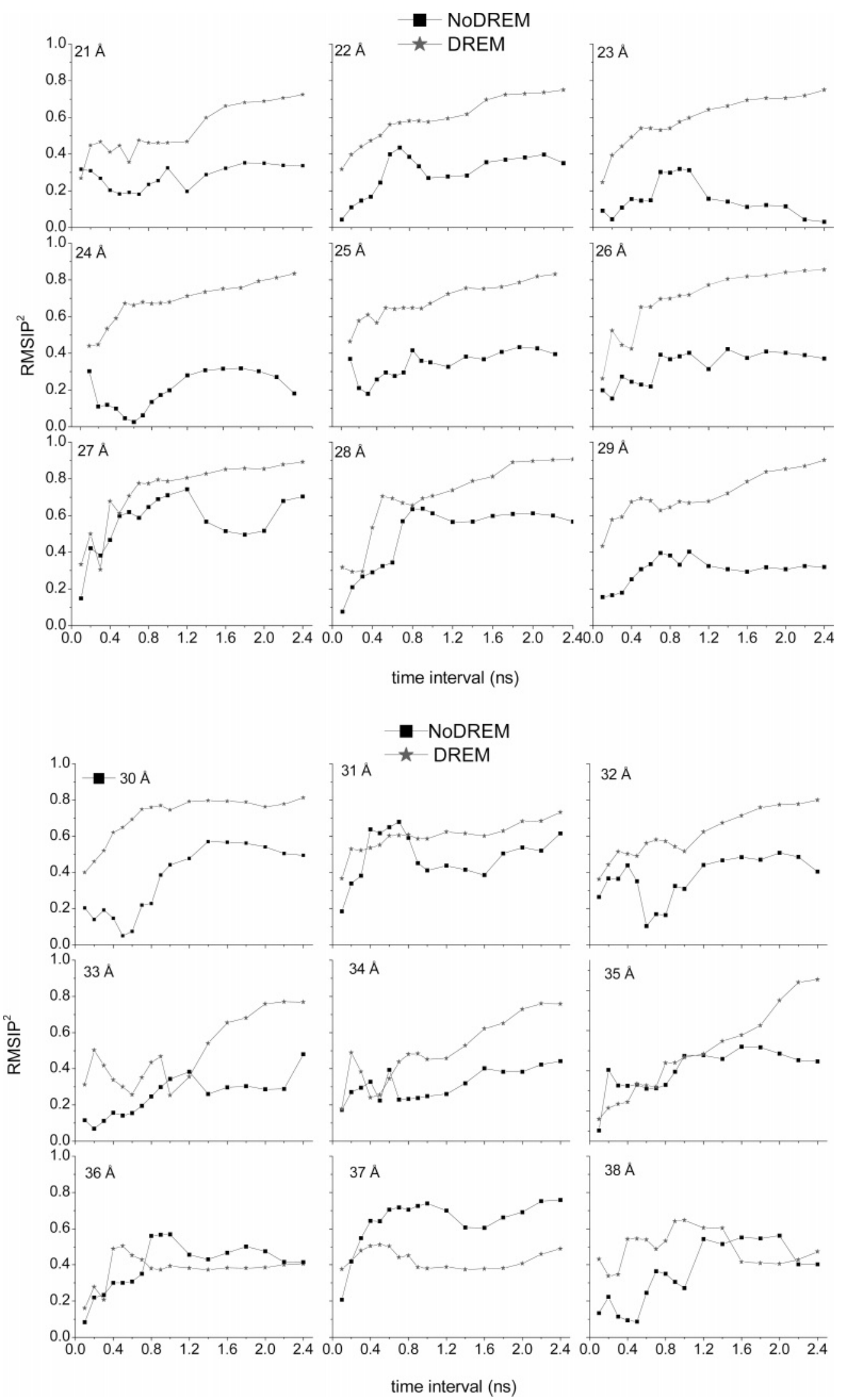

Figure 5. RMSIP convergence test (see eq 2.4). RMSIP ${ }^{2}$ of the subspace spanned by the first two modes for time intervals in the first half overlapped with time intervals from the second half of the last $5 \mathrm{~ns}$ of the trajectory.

This absence of sufficient decay of the time autocorrelation in the NoDREM simulation precludes the estimation of a relaxation time. For mode two of the DREMB (Figure 6b), the $39 \AA$ system does not approach zero very quickly, while all the other systems do approach zero very rapidly. For the NoDREM, again, the decay to zero is slow. Thus, we conclude that compared to NoDREM, DREMB significantly reduces the decay time of the time autocorrelation functions. Faster decay of the mode time autocorrelation functions leads to a reduction in the variance of the PCA covariance matrix, accelerating the convergence of 
(a)

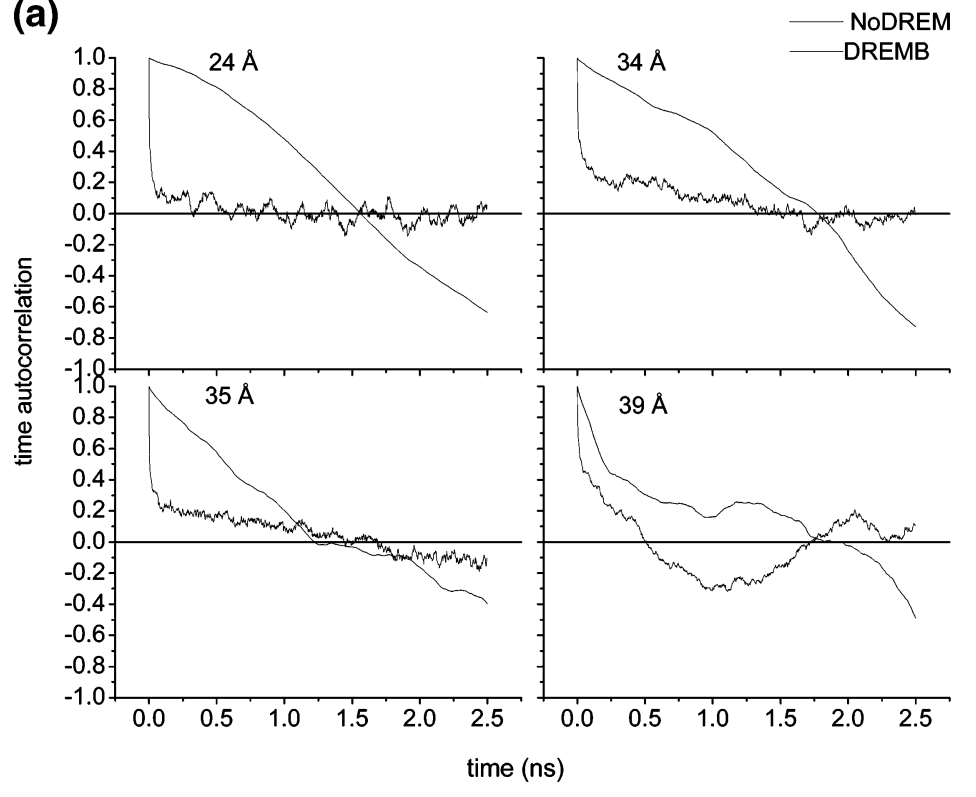

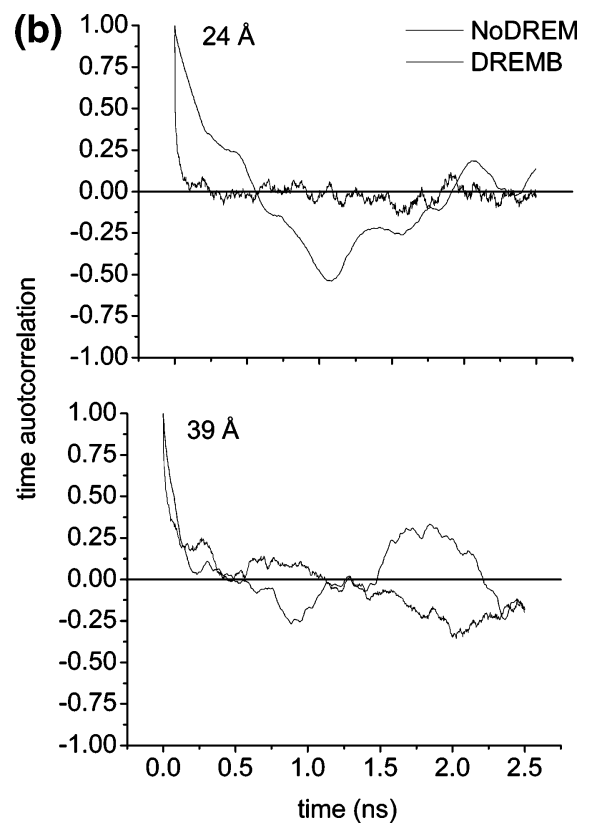

Figure 6. (a) DREMB and NoDREM (no exchange attempts) time autocorrelations for PCA mode one, for $24,34,35$, and $39 \AA$ A. The DREMB $24 \AA$ A plot is representative of the fast decay DREMB systems. The DREMB systems with equilibrium window distances of 34,35 , and $39 \AA$ A show slower decay. The NoDREM systems for all equilibrium window distances exhibit slow decay behavior. (b) The DREMB and NoDREM time autocorrelations for PCA mode two, for 24 and $39 \AA$ A, respectively. The $24 \AA$ plot is representative of the fast decay DREMB systems. The $39 \AA$ DREMB system is the only one with slower decay. The NoDREM decays are slow for all equilibrium window distances.
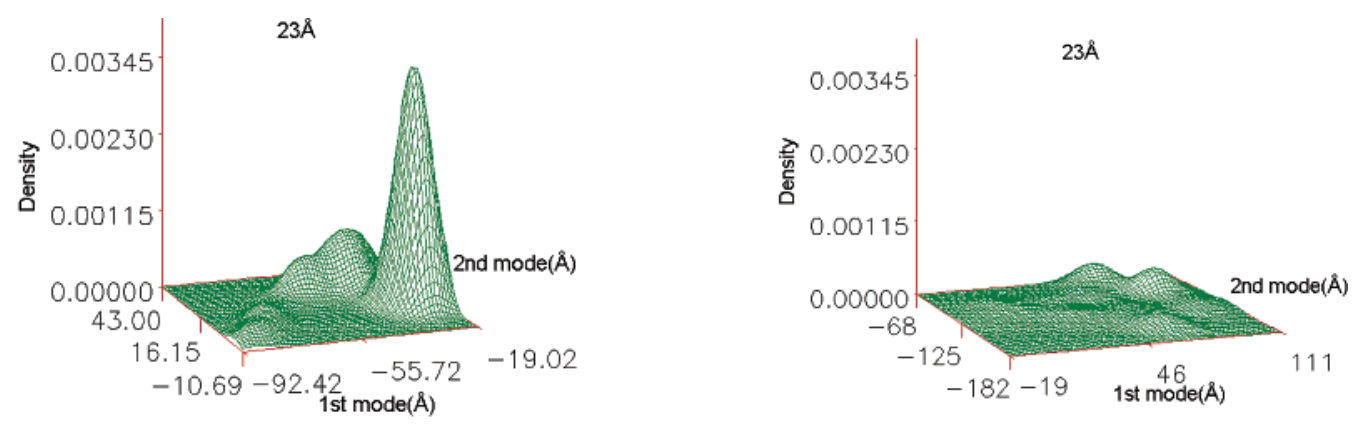

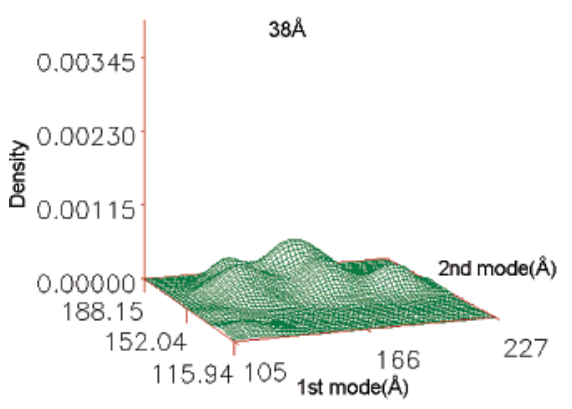

(a)

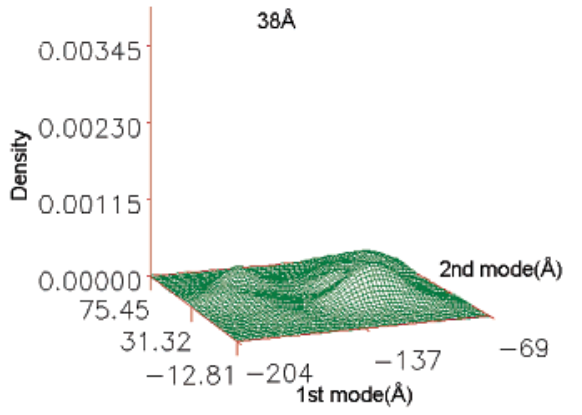

(b)

Figure 7. Probability density in the plane spanned by the first two PCA modes for the 23 and $38 \AA$ systems. The $23 \AA$ result is representative of all the systems other than the $38 \AA$ system. (a) NoDREM. (b) DREMB. The $38 \AA$ NoDREM and DREMB systems occupy almost the same area, while for all other systems, the DREMB probability is much more spread out than that for the NoDREM.

the covariance matrix. ${ }^{53}$ The presence of slow decays in the NoDREM time autocorrelation functions for 34,35 , and $39 \AA$ could come from the slow relaxation of degrees of freedom other than the reaction coordinate, where other free energy barriers exist.

A complementary way to study the efficiency of the DREM is to compare the extent of the configuration space sampled by DREMB and NoDREM. Because the essential space of the PCA takes up most of the protein motion, it is natural to examine the volume of this essential space. This is the strategy used by Zhang, ${ }^{55}$ as well as Sanbonmatsu and Garcia, ${ }^{56}$ in TREM simulations. They found that their systems explored more space, based on the first two PCA modes, than the space explored by normal MD, when comparable total simulation times are used for the comparison. We use the kernel density estimation method with a Gaussian kernel ${ }^{57}$ to estimate the probability density in the plane spanned by the first two modes. Figure 7 presents the typical probability density of the system in the plane spanned by the first two modes of the PCA analysis. We deliberately make the scale in the probability density axis the same, while we set the scales of the other axes according to their respective ranges to contrast the differences between the DREMB and 
NoDREM results. The probability density of the system with equilibrium distance $24 \AA$ is representative of the other 17 systems, with the exception of the one at $38 \AA$. The NoDREM density plots are high and sharp, while the DREMB plots are quite flat. The difference is striking. Because the integral of the probability density over the whole plane is unity, the flatness in the DREMB density plots indicate that the areas explored in the DREMB simulation is much greater than the areas explored in the NoDREM simulation. The one exception is for the system at $38 \AA$; it covers about the same area in both the DREMB and NoDREM simulations.

Sanbonmatsu and Garcia found ${ }^{56}$ in their TREM study that the space spanned by the first two modes is about five times greater than that in the MD simulation with comparable aggregated simulation time. There are a number of ways of estimating the spanning space. Here, first, in an aggressive way, we calculate the space by calculating the area of the rectangle formed by the ranges of the mode one and two trajectories. However, this range method is very prone to errors due to outliers and low probability regions in the data, and therefore, this range estimation may not be accurate. To be more conservative, we also introduce another way to estimate the space explored by the simulation that we call the $p \%$ most probable volume. It is the volume in which the most probable events take place with probability $p \%$. For example, the $\mathrm{p} \%$ most probable volume for the standard normal distribution is the length starting from the $(50-p / 2) \%$ quantile and ending with the $(50+p / 2) \%$ quantile, noting that the standard normal distribution decreases symmetrically from its maximum at zero. A rough algorithm is used to estimate this volume: First, grid the space into cells using a particular resolution. Second, distribute all the points to the cells in the grid. Third, sort the cells in descending order according to the number of points they contain. Fourth, starting with the cell with the greatest number of points, add up the number of points in the cells until the accumulated number of points is equal to $N p \%$, where $N$ is the total number of points. The number of cells counted multiplied by the volume of the unit cell then is the $p \%$ most probable volume. In principle, this algorithm can be applied to find the volume in space for any dimension; however, the number of points required to maintain the accuracy of the volume increases roughly exponentially. Here, we only apply it to the essential subspace formed by the plane spanned by the first two PCA modes. To make the results from the DREMB and NoDREM comparable, we make the average number of points over the cells the same. Also, to make sure that the method is reliable, two estimations are made for each DREMB and NoDREM simulation, with two different average numbers of points in the cell. Figure 8 displays the $75 \%$ most probable area spanned by the first two PCA modes in DREMB and NoDREM, with the average number of points in a cell 4 and 8 . The two estimations using different average number points in a cell are very similar, confirming the reliability of our method. With the exception of the equilibrium window distance $38 \AA$, where the NoDREM is slightly bigger than the DREMB, the area explored by DREMB is bigger than the area explored by NoDREM. This result is consistent with the density plots in Figure 7. In Table 4, we present ratios of areas under the first two PCA modes of DREMB to NoDREM, calculating both in the aggressive way of using rectangles formed by the two ranges in modes one and two, and in the conservative way using the $75 \%$ most probable volume. The system with equilibrium distance $29 \AA$ has the largest ratio of 7 , while the system with equilibrium distance $39 \AA$ has the smallest ratio of 1.7 . So, we conclude

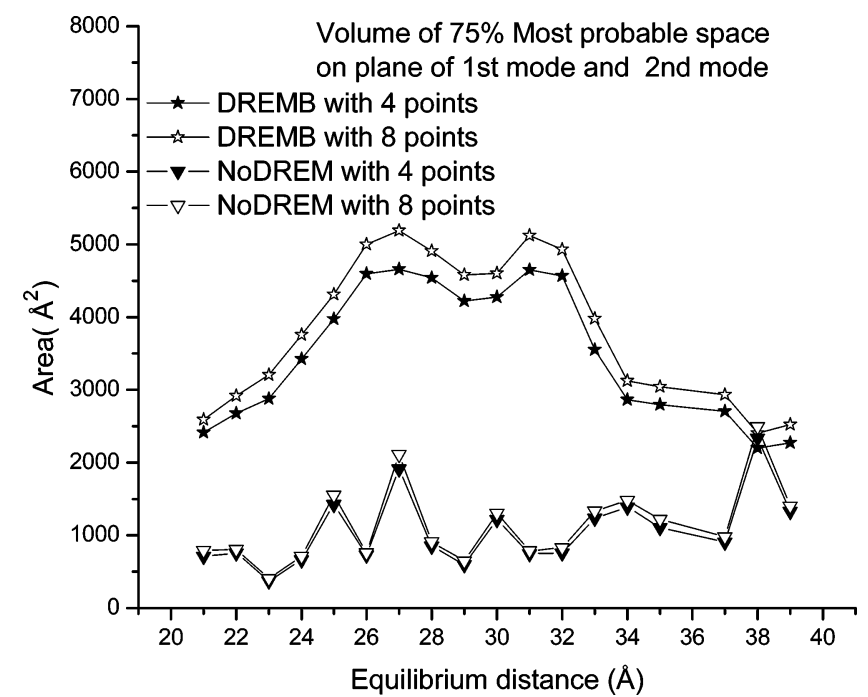

Figure 8. The $75 \%$ most probable area spanned by the first two PCA modes in the DREMB and NoDREM simulations. The areas occupied by the DREMB simulations are, with the exception of the $38 \AA$ equilibrium window distance, considerably greater than in the NoDREM simulation.

TABLE 4: Ratios of Areas Spanned by the First Two Modes of the DREMB and NoDREM Simulations

\begin{tabular}{cccc}
\hline potential index & ratio $^{a}$ & ratio $^{b}$ & ratio $^{c}$ \\
\hline 1 & 3.02 & 3.40 & 3.28 \\
2 & 2.93 & 3.55 & 3.61 \\
3 & 3.77 & 7.74 & 7.95 \\
4 & 5.57 & 5.17 & 5.30 \\
5 & 2.97 & 2.79 & 2.78 \\
6 & 7.86 & 6.32 & 6.57 \\
7 & 4.27 & 2.43 & 2.46 \\
8 & 5.55 & 5.33 & 5.38 \\
9 & 8.18 & 7.09 & 7.11 \\
10 & 4.98 & 3.52 & 3.53 \\
11 & 7.65 & 6.20 & 6.51 \\
12 & 5.01 & 6.03 & 5.95 \\
13 & 2.91 & 2.87 & 2.98 \\
14 & 3.56 & 2.06 & 2.11 \\
15 & 3.33 & 2.53 & 2.50 \\
16 & 3.11 & 2.99 & 3.00 \\
18 & 1.35 & 0.94 & 0.96 \\
19 & 3.61 & 1.71 & 1.80
\end{tabular}

${ }^{a}$ Based on the ranges of PCA modes 1 and 2. ${ }^{b}$ Based on the $75 \%$ most probable volume (4 pts) of PCA modes 1 and $2 .{ }^{c}$ Based on the $75 \%$ most probable volume ( 8 pts) of PCA modes 1 and 2 .

that the DREMB simulation has explored considerably more space than the NoDREM simulation, except for the system with equilibrium distance $38 \AA$.

In summary, comparing the DREMB and NoDREM simulations with the same time length and same initial conditions (see Methods Section), the DREMB's essential space, occupying at least $58 \%$ of the total fluctuation, converges faster than the NoDREM's essential space, occupying at least $49 \%$ total of the total fluctuation. In terms of time autocorrelations, the DREMB systems decay to zero much faster than the NoDREM systems. At the same time, the DREMB simulation explores considerably more space in the essential space. These three comparisons provide strong evidence that the DREMB performs sampling more efficiently than does the NoDREM simulation.

PMF Calculation and Biological Implications. The WHAM provides a convenient, statistically based approach to combine trajectory data from multiple windows with their different biasing potentials and obtain the PMF along a reaction coordinate. Here, we use it to calculate the PMF along our 


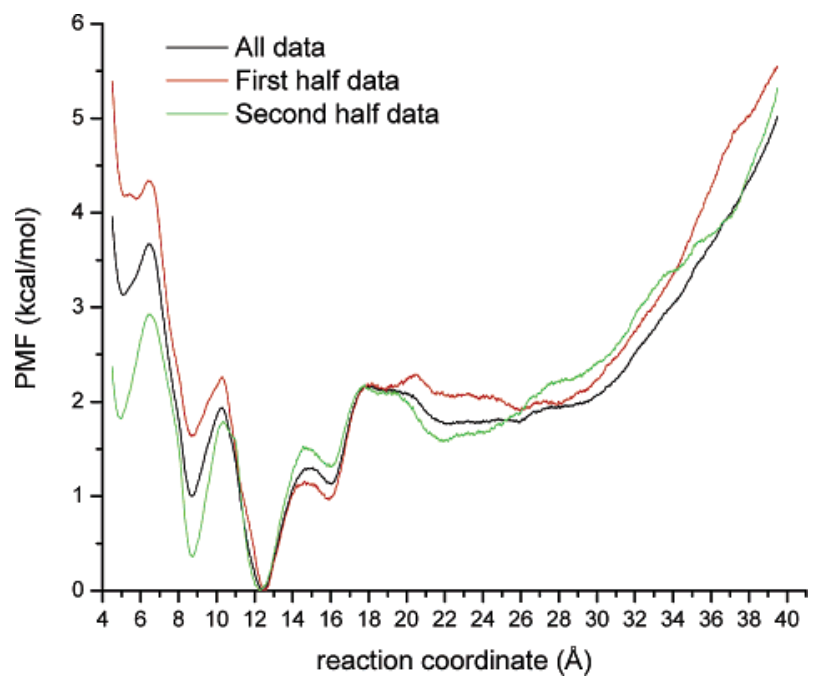

Figure 9. PMF for the first half, second half, and all of the trajectory data.

selected reaction coordinate, the distance between the mass centers of residues 55 and 169 , which are the residues that were labeled in the energy transfer experiments ${ }^{7}$ and serve as a monitor of the Amp-bd domain-to-core distance. To make sure of the convergence of the PMF, we calculated the PMF for the time corresponding to the first half and the second half of the trajectory for the DREMA and DREMB data. In addition, two trajectories (7 ns with the first ns discarded) of equilibrium distance of 19 and $20 \AA$ of the DREMP simulation are used to connect the DREMA and DREMB simulations. Those two trajectories are not in the problematic regions and have good converging distributions along the reaction coordinate for the different time intervals.

The energy transfer experiments by Sinev et al. ${ }^{7}$ show that AKE has an average reaction coordinate value of $31.0 \AA$ for the free form, $23.2 \AA$ for the AMP-bound form, $18.7 \AA$ for the $\mathrm{Mg}^{2+}$-ATP bound form, and $11.5 \AA$ for the $\mathrm{AP}_{5} \mathrm{~A}$ (a mimic of the tertiary complex) bound form. The reaction coordinate value is $28.7 \AA$ in the free form X-ray structure and $11.5 \AA$ in the $\mathrm{AP}_{5} \mathrm{~A}$-bound $\mathrm{X}$-ray structure. ${ }^{1}$ Figure 9 displays the PMF. The PMF from 10 to $39 \AA$ converges very well, with some discrepancy for reaction coordinate values less than $10 \AA$. The lowest PMF value along the reaction coordinate occurs at 12.4 $\AA$. This distance is very close to the distance in the $\mathrm{AP}_{5} \mathrm{~A}$-bound form, and below, we show that this most stable state is similar to the closed form. Note that there is no barrier when one goes from 39 to $10 \AA$, and all the reaction coordinate distances that correspond to the open, AMP-bound, and $\mathrm{Mg}^{2+}$-ATP-bound forms have at least a $2 \mathrm{kcal} / \mathrm{mol}$ difference with the minimum PMF value at $12.4 \AA$. This free energy difference translates to a ratio of occupation probabilities of about 28. However, noting that the PMF from 18 to $30 \AA$ is very flat compared to the PMF around $12 \AA$, it is more appropriate to make comparisons based on integrating the corresponding probability densities over reaction coordinate values. Thus, we integrate the probability density over the ranges 18 to $39 \AA$ and 4 to $18 \AA$. The ratio of the second to the first integral is 3.88 , which means that, if we consider conformations with reaction coordinate range from 18 to $39 \AA$ as one state and 4 to $18 \AA$ as another state, then the free energy difference between these two states is only 0.81 $\mathrm{kcal} / \mathrm{mol}$. The flatness of the PMF in the 18 to around $30 \AA$ range is an indication of a large entropic contribution in this region. Distances larger than $\sim 30 \AA$ (the X-ray open form reaction coordinate distance is $28.7 \AA$ ) may correspond to

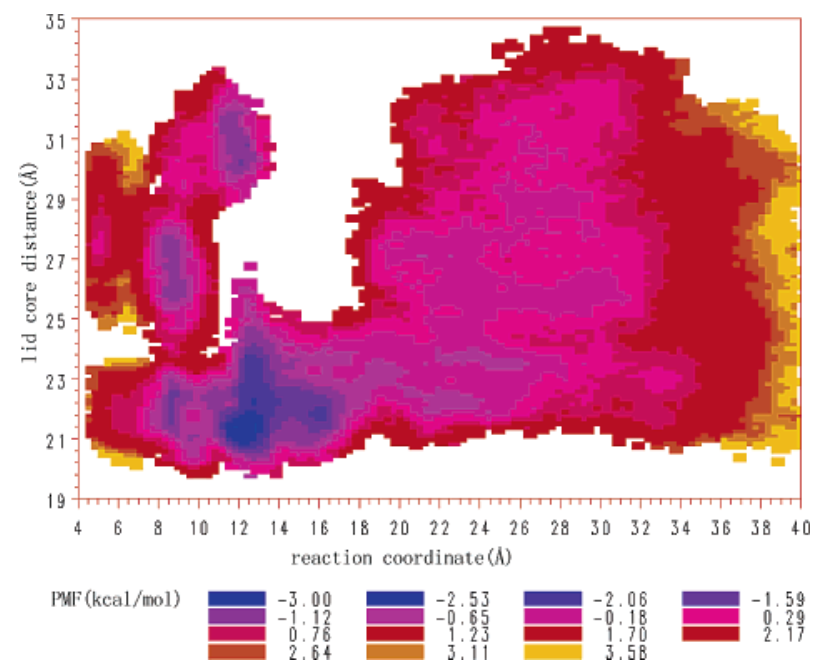

Figure 10. PMF in the plane spanned by the lid-to-core mass center distance and the reaction coordinate.

configurations that are becoming so "stretched" that their probabilities are decreasing due to decreasing entropic and increasing energetic components of the free energy. The PMF result is consistent with that found in the energy transfer experiment, ${ }^{7}$ where the free form does sample a very large number of conformations, as indicated by the large dispersion found around the average reaction coordinate value.

The energy transfer experiment ${ }^{7}$ does not provide information about how the lid reacts to the changes in the reaction coordinate distance. The reaction coordinate monitors the Amp-bd-to-core distance. But, when AKE closes, there is also a large motion of the lid relative to the core, as is evident by comparing the open and closed X-ray structures. In particular, the distance between the mass centers of the lid and core is $29.8 \AA$ in the free form and $20.3 \AA$ in the $\mathrm{AP}_{5} \mathrm{~A}$-bound form $\mathrm{X}$-ray structures. To explore this issue, we calculate $\operatorname{PMF}(x, r)$, the twodimensional PMF in the plane spanned by the reaction coordinate, $r$, and the mass center distance between the lid and core, $x$. Figure 10 displays this two-dimensional PMF. The most stable conformation has a lid-to-core mass center distance of $\sim 20.5$ $\AA$ and a reaction coordinate of $\sim 12.0 \AA$, which are very close to the $\mathrm{AP}_{5} \mathrm{~A}$-bound form distances. Note also that, from 18 to $30 \AA$ of the reaction coordinate, the PMF is not only flat along the reaction coordinate but also along the mass center lid-tocore distance. Most interestingly, in this region, the lid can fluctuate from the distance found for the free form $(\sim 30 \AA)$ to the distance found for the $\mathrm{AP}_{5} \mathrm{~A}$-bound form $(\sim 20 \AA)$ in the $\mathrm{X}$-ray data. This flatness in the lid-to-core region can compensate for the high PMF in the reaction coordinate direction relative to the region around the minimum of $12 \AA$. If one starts with the largest value of the reaction coordinate and decreases it, first, the lid can take on any conformation between the closed and open forms. Then, when the reaction coordinate reaches near $18 \AA$, the fluctuations of the lid-to-core mass center distance become smaller. When the reaction coordinate decreases further, these fluctuations get even smaller. Finally, when the reaction coordinate is around $12 \AA$, the lid is mostly in a closed form, with a relatively small proportion open. Along the lid-to-core direction, there is a substantial free energy barrier separating these closed and open lid conformations.

The induced closure hypothesis that was proposed for AKE is based on the static X-ray structures of apo and various $\mathrm{AKE}^{1}$ complexes where, in the free form, AKE has an open lid and an open Amp-bd site. Then, when AMP binds, the lid and Ampbd domains both close a little and, when $\mathrm{AP}_{5} \mathrm{~A}$ binds, the lid 
and Amp-bd domains are completely closed. The above results show that there is an alternative perspective, which does not contradict the results of the X-ray based induced view. Instead of a completely open conformation (both Amp-bd and lid open) in the free form, in our simulation, AKE can also exist in a completely closed conformation (both Amp-bd and lid closed). Thus, even in the absence of ligands, AKE can sample conformations similar to the completely closed form that is found in the ternary $\left(\mathrm{AP}_{5} \mathrm{~A}\right)$ complex. When the reaction coordinate is in the range of the open form of the Amp-bd domain relative to the core, AKE also can sample multiple lid conformations, ranging between the closed- and open-lid conformers and, as the Amp-bd distance decreases, the lid-tocore distance decreases and its fluctuations are reduced. Furthermore, the existence of multiple conformations in the free form is consistent with NMR experiments ${ }^{5}$ and also with the kinetic studies. ${ }^{2}$

Another perspective on the conformations sampled is obtained by constructing a two-dimensional histogram, using for one coordinate, $x$, the trajectory RMSD relative to the closed $\mathrm{X}$-ray form and for the other, $y$, the trajectory RMSD relative to the open X-ray form. To do this, we first calculate the unbiased density $\rho^{\mathrm{u}}(x, y)$ in the $x-y$ plane based on an extension of the WHAM method ${ }^{51,52}$ that is developed in the Methods Section. The density is then converted to a $\operatorname{PMF}$ according to $\operatorname{PMF}(x, y)$ $=-k_{\mathrm{B}} T \ln \rho^{\mathrm{u}}(x, y)$. It turns out (data not shown) that the most populated conformations in the $x-y$ plane are those around the closed-form X-ray structure. ${ }^{58}$ In Figure 11 , we display a snapshot from the system with window equilibrium distance of $12 \AA$ to show how close it is to the closed X-ray structure. The CA RMSD of the snapshot fit on the closed-form core is 4.0 $\AA$, while the CA RMSD fit on the open-form core is $7.0 \AA$.

\section{Conclusions}

In this paper, we showed that the DREM is much more efficient in sampling configuration space than a normal MD simulation with umbrella sampling. To demonstrate this, we focused on the first two PCA modes that represent a substantial fraction of the protein fluctuations and should correspond to the more directed parts of the protein motion. The DREM has better convergence behavior than the corresponding NoDREM simulation in the PCA subspace spanned by the first two vectors. Indeed, the DREM greatly reduced the time autocorrelation of the trajectories of the first and second mode displacements relative to the NoDREM simulations. From the perspective of a given system (window potential), the visits from other replicas introduce new configurations that are relatively far from the current configuration and will reduce the time correlation much faster than in the absence of exchanges. Consequently, the convergence of the covariance matrix on which the PCA relies is accelerated. The enhanced sampling of the DREM method is also reflected in the larger volume explored in the subspace of the first two PCA modes relative to the NoDREM simulation. Over the time interval of the simulation, the convergence toward unity of the RMSIP overlap measure is excellent for the DREM, indicating that reliable predictions can be made for the large conformational transformation of AKE under study.

A comparison of the one- (Figure 9) and two-dimensional PMFs (Figure 10) is instructive. The DREMA simulation for the inner range $(r \sim 5-18 \AA)$ required a more elaborate parametrization of the window functions than did the DREMB simulation for the outer range $(r \sim 21-39 \AA)$ of the reaction coordinate. Not surprisingly, once the Amp-bd domain has been pulled in substantially, with the concomitant decrease in the

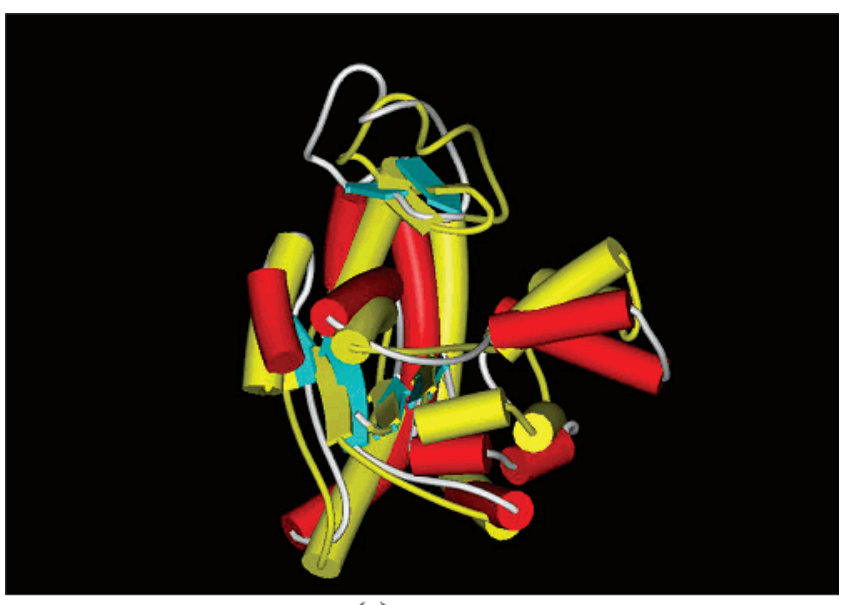

(a)

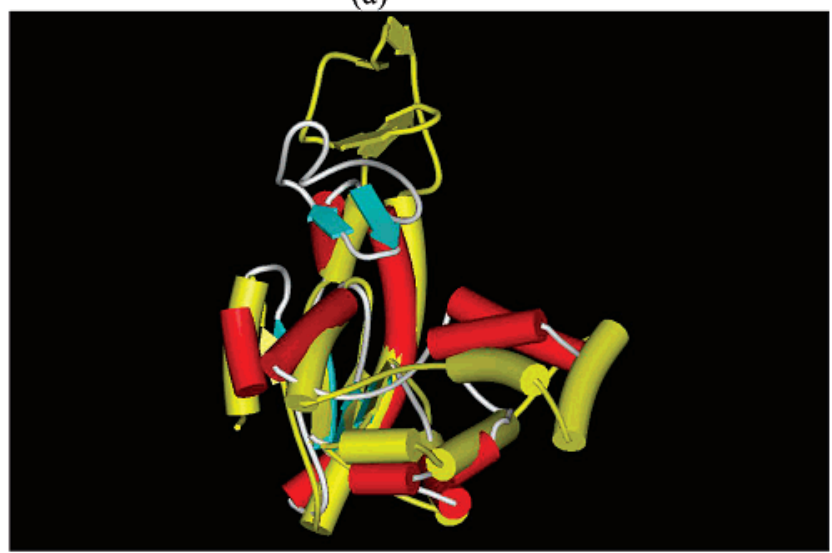

(b)

Figure 11. Representative snapshot from the system with equilibrium window distance $12 \AA$ superposed on (a) the closed-form X-ray structure (yellow trace) and (b) the open form X-ray structure (yellow trace). In each case, the snapshot is fit on the core of the X-ray structure. The snapshot resembles the closed form much more closely than the open form, indicating that the apo form can fluctuate to a near-closed conformation.

lid-to-core distance, the resulting increase in atom interactions requires many subtle adjustments. That is one possible contributor to the greater discrepancy in the $\operatorname{PMF}(r)$ obtained using the first and second half of the trajectory data for this inner region evident in Figure 9. Another potential contributing factor, revealed in Figure 10, is the complex structure of the $\operatorname{PMF}(x, r)$ for $r<10 \AA$, with the well-separated conformers in the lidto-core direction for both the Amp-bd-to-core distances of $\sim 12$ and $\sim 8.5 \AA$. It is encouraging that the DREM can provide reasonable results in this difficult-to-sample region. The configurational updates from exchanges of systems with different window potentials also introduce information from different lidto-core distances.

AKE can sample multiple conformations in the free form. The PMF along the reaction coordinate (Figure 10) shows a broad, flat region for larger values and a "hole" around $12 \AA$, a distance that corresponds well with the Amp-bd core distance in the closed, $\mathrm{AP}_{5} \mathrm{~A}$-bound X-ray structure. The Amp-bd domain is most likely sampling a large range of conformations at these larger reaction coordinate distances, indicating a substantial entropic component to this flat free energy surface. Our previous simulations on free form AKE that did not use a reaction coordinate method did show (from a study of the PCA modes) a tendency to fluctuate in the closing direction, but evidently there are significant energy barriers to the closing motion that could not be overcome in a conventional MD simulation. While 
the small free energy difference $(\sim 0.8 \mathrm{kcal} / \mathrm{mol})$ favoring "closed" (4-18 $)$ ) versus "open" $(18-39 \AA)$ that we find is consistent with the experimental data, it must be stressed that, allowing for the quality of MD force fields and the differences between experimental and simulation systems, it is only safe to conclude that there is some reasonable fraction of "closed" states.

Examination of conformations along the reaction coordinate trajectory showed that, in addition to ones similar to the $\mathrm{AP}_{5} \mathrm{~A}$ bound conformation, there are conformations in which the lidcore distance ranges from the corresponding distance in the free form $\mathrm{X}$-ray structure to the one in the $\mathrm{AP}_{5} \mathrm{~A}$-bound $\mathrm{X}$-ray structure. The two-dimensional PMF in the reaction coordinate and lid-core distance variables (Figure 10) shows that, as the reaction coordinate distance decreases, AKE's lid progressively changes its motion from readily fluctuating between its open and closed conformations toward more constrained fluctuations until it mainly stays in the closed-form conformation when the reaction coordinate is around $12 \AA$. It is interesting that, in the simulation protocol where the Amp-bd-to-core distance is reduced gradually, the lid-to-core distance responds to this reduction by also decreasing, and does so in a manner that eventually produces conformations similar to the closed-form $\mathrm{X}$-ray structure. Furthermore, as these distances decrease, the range of lid-to-core distances sampled decreases, in agreement with the reduction in fluctuations as successive ligands are bound in the energy transfer experiments. The correlation between these distances does not contradict the random bi bi mechanism suggested for AKs. ${ }^{59}$ That is, once ligand free closed conformations are sampled, the ligands can then bind in random order, and both are required for the final catalytically competent structure.

The open-to-closed transition in AKE has been studied by methods that are generalizations of the elastic network model (ENM) whereby the path between open and closed forms is obtained by mixing these two endpoints. ${ }^{21,20}$ Both studies conclude that the lid and Amp-bd domains can rearrange without a great (free) energy penalty, while the core is quite stable during this transition. Maragakis and Karplus, ${ }^{21}$ starting from the open form, find that first mainly the lid closes and then the Amp-bd domain closes. They attribute the order to the small energy cost of moving the lid, with its flexible hinge connections to the core domain. They identify hinges for the open-to-closed pathway that are similar to those found using the open and closed X-ray structures. Because our simulation uses a reaction coordinate, the Amp-bd core distance, we cannot obtain an order of closing. Note that our result does not rely on using a specified closed form endpoint; the closed form we obtain emerges just from the distance restraint on the Amp-bd-to-core distance. We determined the hinges between the open-form X-ray structure and the closed structure snapshot displayed in Figure 11, using the DynDom program. ${ }^{60}$ The results show that there are hingebending regions around the beginning (residues 117-123) and end (residues 153-167) of the lid domain and around the beginning (residues 26-29) and end (residues 64-66) of the Amp-bd domain. These are quite close to those found in the ENM work $^{21}$ that used DynDom and similar to those found by other methods using open (beef heart) and closed (AKE) X-ray structures. $^{10}$

The ease with which AK can sample a large conformational space is supported by the MD and ENM approaches. In this regard, a recent investigation of chimeric forms of adenylate kinase that are constructed by switching regions of mesophilic and thermophilic varieties showed that the core is mainly involved in thermal stability while the lid and Amp-bd domains are not. ${ }^{61}$ In the DREM simulations, the core structure is well maintained at all stages along the reaction coordinate, indicating the stability of the core during the large-scale rearrangements of lid and Amp-bd domains, which is consistent with the chimera results. Furthermore, in a previous MD simulation of apo AKE, ${ }^{18}$ we found that raising the simulation temperature induced an increase in the mobility of the lid and Amp-bd domains while the core stayed stable.

The scenario presented above provides an attractive alternative to the induced fit hypothesis, where ligand binding is required for closing AKE. Instead, what we find is that the apo form of AKE can sample a broad range of conformational states, some of which resemble the final closed form. These conformations may be more advantageous for ligand binding by requiring smaller protein rearrangements (with a reduced energetic requirement) than would be necessary in the more open forms. Of course, in the process of binding ligands, an entropic penalty from restricting the space available to the ligands must be compensated for by the formation of ligand-protein interactions. These final rearrangements must occur even if the protein is in a state resembling the ligand-bound closed form.

Acknowledgment. This work is supported by the NIH (grant no. GM62790). The simulations were carried out on the Michigan Center for Biological Information Linux cluster at Michigan State University.

\section{Appendix A}

The average acceptance ratio is defined as

$$
p_{\text {acc }}=\int \mathrm{d} \mathbf{R}_{0} \int \mathrm{d} \mathbf{R}_{1} P_{0}\left(\mathbf{R}_{0}\right) P_{1}\left(\mathbf{R}_{1}\right) \alpha\left(\mathbf{R}_{0} \mathbf{R}_{1} \rightarrow \mathbf{R}_{1} \mathbf{R}_{0}\right)
$$

with the transition probability satisfying the detailed balance condition of eq 2.1. For a Metropolis Monte Carlo transition probability,

$$
\begin{aligned}
\alpha\left(\mathbf{R}_{0} \mathbf{R}_{1} \rightarrow \mathbf{R}_{1} \mathbf{R}_{0}\right) & =1 \quad(\Delta \leq 0) \\
& =\exp (-\Delta) \quad(\Delta>0)
\end{aligned}
$$

with, for a general HREM simulation,

$$
\Delta\left(\mathbf{X}_{i} \mathbf{X}_{j} \rightarrow \mathbf{X}_{j} \mathbf{X}_{i}\right)=\beta\left[\left(E_{i}\left(\mathbf{X}_{j}\right)+E_{j}\left(\mathbf{X}_{i}\right)\right)-\left(E_{i}\left(\mathbf{X}_{i}\right)+E_{j}\left(\mathbf{X}_{j}\right)\right)\right]
$$

eq A.1 can be resolved as

$$
\begin{aligned}
p_{\text {acc }}= & \underset{\alpha\left(\mathbf{R}_{0} \mathbf{R}_{1} \rightarrow \mathbf{R}_{1} \mathbf{R}_{0}\right)=1}{\iint_{0}} \mathrm{~d} \mathbf{R}_{0} \mathrm{~d} \mathbf{R}_{1} P_{0}\left(\mathbf{R}_{0}\right) P_{1}\left(\mathbf{R}_{1}\right)+ \\
& \quad \iint_{\alpha\left(\mathbf{R}_{0} \mathbf{R}_{1} \rightarrow \mathbf{R}_{1} \mathbf{R}_{0}\right)<1} \mathrm{~d} \mathbf{R}_{0} \mathrm{~d} \mathbf{R}_{1} P_{0}\left(\mathbf{R}_{0}\right) P_{1}\left(\mathbf{R}_{1}\right) \alpha\left(\mathbf{R}_{0} \mathbf{R}_{1} \rightarrow \mathbf{R}_{1} \mathbf{R}_{0}\right)
\end{aligned}
$$

The second integral's area restriction can be expressed in terms of the definitely accepted region with the use of the detailed balance condition since

$$
\begin{array}{r}
\iint_{\left.\mathbf{R}_{0} \mathbf{R}_{1} \rightarrow \mathbf{R}_{1} \mathbf{R}_{0}\right)<1} \mathrm{~d} \mathbf{R}_{0} \mathrm{~d} \mathbf{R}_{1} P_{0}\left(\mathbf{R}_{0}\right) P_{1}\left(\mathbf{R}_{1}\right) \alpha\left(\mathbf{R}_{0} \mathbf{R}_{1} \rightarrow \mathbf{R}_{1} \mathbf{R}_{0}\right) \\
=\underset{\alpha\left(\mathbf{R}_{0} \mathbf{R}_{1} \rightarrow \mathbf{R}_{1} \mathbf{R}_{0}\right)<1}{\int} \mathrm{d} \mathbf{R}_{0} \mathrm{~d} \mathbf{R}_{1} P_{0}\left(\mathbf{R}_{1}\right) P_{1}\left(\mathbf{R}_{0}\right)
\end{array}
$$


Here, we have used the property that the "reverse" transition probability must be unity for this region of integration. Then, interchange of integration variables $\mathbf{R}_{0} \rightarrow \mathbf{X}_{1}, \mathbf{R}_{1} \rightarrow \mathbf{X}_{0}$ in eq A.4 provides

$$
\begin{array}{rl}
\underset{\alpha\left(\mathbf{R}_{0} \mathbf{R}_{1} \rightarrow \mathbf{R}_{1} \mathbf{R}_{0}\right)<1}{\iint_{0}} & \mathrm{~d} \mathbf{R}_{0} \\
= & \int \mathbf{R}_{1} P_{0}\left(\mathbf{R}_{1}\right) P_{1}\left(\mathbf{R}_{0}\right) \\
& \iint_{\alpha\left(\mathbf{X}_{1} \mathbf{X}_{0} \rightarrow \mathbf{X}_{0} \mathbf{X}_{1}\right)<1} \mathrm{~d} \mathbf{X}_{0} \mathrm{~d} \mathbf{X}_{1} P_{0}\left(\mathbf{X}_{0}\right) P_{1}\left(\mathbf{X}_{1}\right) \\
= & \iint_{\alpha\left(\mathbf{X}_{0} \mathbf{X}_{1} \rightarrow \mathbf{X}_{1} \mathbf{X}_{0}\right)=1} \mathrm{~d} \mathbf{X}_{0} \mathrm{~d} \mathbf{X}_{1} P_{0}\left(\mathbf{X}_{0}\right) P_{1}\left(\mathbf{X}_{1}\right)
\end{array}
$$

Adding this to the first integration region result in eq A.3 yields eq 2.7 .

A generalization of eq 2.7 can be devised that may be of use for certain types of replica exchange rules. The above equation will not hold if there exists a finite area where $\Delta=0$ in the Cartesian product space $\mathbf{X}_{0} \times \mathbf{X}_{1}$. When $\Delta=0, \alpha\left(\mathbf{X}_{0} \mathbf{X}_{1} \rightarrow\right.$ $\left.\mathbf{X}_{1} \mathbf{X}_{0}\right)=\alpha\left(\mathbf{X}_{1} \mathbf{X}_{0} \rightarrow \mathbf{X}_{0} \mathbf{X}_{1}\right)=1$. For example, in a temperature exchange method, where two systems have the same temperature, the system must exchange with probability 1 on every attempt. So, according to eq A.5, the acceptance ratio from method 2 will double the acceptance ratio of method 1 . This problem comes from the fact that, under the whole area formed by Cartesian product $\mathbf{X}_{0} \times \mathbf{X}_{1}, \Delta \equiv 0$. Then the area where $\Delta$ $=0$ will double count the probability. The error comes from the reasoning in eq A.5 that the area where $\alpha\left(\mathbf{X}_{1} \mathbf{X}_{0} \rightarrow \mathbf{X}_{0} \mathbf{X}_{1}\right)$ $<1$ is equivalent to the area where $\alpha\left(\mathbf{X}_{0} \mathbf{X}_{1} \rightarrow \mathbf{X}_{1} \mathbf{X}_{0}\right)=1$. Apparently, if there exists a finite area where $\alpha\left(\mathbf{X}_{0} \mathbf{X}_{1} \rightarrow \mathbf{X}_{1} \mathbf{X}_{0}\right)$ $=\alpha\left(\mathbf{X}_{1} \mathbf{X}_{0} \rightarrow \mathbf{X}_{0} \mathbf{X}_{1}\right)=1$, then the area will be double counted. A more general form for $p_{\text {acc }}$ based on this reasoning is

$$
\begin{array}{r}
p_{\mathrm{acc}}=2 \int_{\Delta\left(\mathbf{X}_{0} \mathbf{X}_{1} \rightarrow \mathbf{X}_{1} \mathbf{X}_{0}\right) \leq 0} \mathrm{~d} \mathbf{X}_{1} \mathrm{~d} \mathbf{X}_{0} P_{0}\left(\mathbf{X}_{0}\right) P_{1}\left(\mathbf{X}_{1}\right)- \\
\int_{\Delta\left(\mathbf{X}_{0} \mathbf{X}_{1} \rightarrow \mathbf{X}_{1} \mathbf{X}_{0}\right)=0} \mathrm{~d} \mathbf{X}_{1} \mathrm{~d} \mathbf{X}_{0} P_{0}\left(\mathbf{X}_{0}\right) P_{1}\left(\mathbf{X}_{1}\right)
\end{array}
$$

In the case of the DREM and the TREM, the second part of this equation is zero because there is no area where $\Delta\left(\mathbf{X}_{0} \mathbf{X}_{1} \rightarrow\right.$ $\left.\mathbf{X}_{1} \mathbf{X}_{0}\right)=0$, because $\Delta\left(\mathbf{X}_{0} \mathbf{X}_{1} \rightarrow \mathbf{X}_{1} \mathbf{X}_{0}\right)=0$ means a line (one lower dimension in the Cartesian product space $\mathbf{X}_{0} \times \mathbf{X}_{1}$ ) and the probability density function is assumed to be continuous. This issue was also identified in a recent work, and a similar remedy was proposed. ${ }^{50}$

A circumstance where eq A.6 would be necessary would be in a HREM, where the exchange rule parameter $\Delta\left(\mathbf{X}_{0} \mathbf{X}_{1} \rightarrow\right.$ $\mathbf{X}_{1} \mathbf{X}_{0}$ ) would depend on a cutoff energy. That is, an energy threshold would first be evaluated in order to decide if the Hamiltonians for systems 0 and 1 are different. That would lead to a finite area of configuration space where $\Delta\left(\mathbf{X}_{0} \mathbf{X}_{1} \rightarrow \mathbf{X}_{1} \mathbf{X}_{0}\right)$ $=0$ and necessitates the use of eq A.6.

\section{Appendix B}

We want to show that the expected value of the estimated probability that the coordinate values $(s, t)$ lie in the rectangle $(x, y) \times(x+\Delta x, y+\Delta y)$ is unbiased. That is, the average of the estimated probability is the true value when it is constructed according to the window method we used in the Results Section. The estimated probability $\hat{P}^{(\mathrm{u})}$ is expressed as

$$
\begin{array}{r}
\hat{P}^{(u)}((s, t) \in(x, y) \times(x+\Delta x, y+\Delta y))= \\
\sum_{w=1}^{N} \sum_{j=1}^{n_{\mathrm{w}}} \frac{I\left(\left(x_{j}^{\mathrm{w}}, y_{j}^{\mathrm{w}}\right) \in(x, y) \times(x+\Delta x, y+\Delta y)\right)}{G\left(r_{j}^{\mathrm{w}}\right)}
\end{array}
$$

where the indicator function is

$$
\begin{aligned}
& I((s, t) \in(x, y) \times(x+\Delta x, y+\Delta y))= \\
& \begin{cases}0 & (s, t) \notin(x, y) \times(x+\Delta x, y+\Delta y) \\
1 & (s, t) \in(x, y) \times(x+\Delta x, y+\Delta y)\end{cases}
\end{aligned}
$$

and

$$
G(r)=\sum_{w=1}^{N} n_{\mathrm{w}} e^{-\beta\left(W_{\mathrm{w}}(r)-f_{\mathrm{w}}\right)}
$$

Here, for window $w, W_{\mathrm{w}}(r)$ is the window function, $n_{\mathrm{w}}$ is the number of points in the window, and $f_{\mathrm{w}}$ is the corresponding free energy. Now, the expected value of the estimated probability is

$$
\begin{aligned}
& E\left[\hat{P}^{(\mathrm{u})}((s, t) \in(x, y) \times(x+\Delta x, y+\Delta y))\right]= \\
& \sum_{w=1}^{N} E\left[\sum_{j=1}^{n_{\mathrm{w}}} \frac{I\left(\left(x_{j}^{\mathrm{w}}, y_{j}^{\mathrm{w}}\right) \in(x, y) \times(x+\Delta x, y+\Delta y)\right)}{G\left(r_{j}^{\mathrm{w}}\right)}\right] \\
& =\sum_{w=1}^{N} n_{\mathrm{w}} E_{\mathrm{w}}\left[\frac{I((s, t) \in(x, y) \times(x+\Delta x, y+\Delta y))}{G(r)}\right] \\
& =\sum_{w=1}^{N} n_{\mathrm{w}} \iiint \frac{I((s, t) \in(x, y) \times(x+\Delta x, y+\Delta y))}{G(r)} \rho_{\mathrm{w}}^{(\mathrm{b})}(s, t, r) \mathrm{d} s \\
& =\iiint \sum_{w=1}^{N} \frac{n_{\mathrm{w}} \rho_{\mathrm{w}}^{(\mathrm{b})}(s, t, r)}{G(r)} I((s, t) \in(x, y) \times(x+\Delta x, y+\Delta y))
\end{aligned}
$$

where $\rho_{\mathrm{w}}^{(\mathrm{b})}(s, t, r)$ is the biased distribution for window $w$.

According to Souaille and Roux, ${ }^{52}$ the WHAM method provides the connection

$$
\sum_{w=1}^{N} \frac{n_{\mathrm{w}} \rho_{\mathrm{w}}^{(\mathrm{b})}(s, t, r)}{G(r)}=\rho^{(\mathrm{u})}(s, t, r)
$$

between the $\rho_{\mathrm{w}}^{(\mathrm{b})}(s, t, r)$ and $\rho^{(\mathrm{u})}(s, t, r)$, the unbiased distribution. Therefore, using eq B.5 in B.4,

$$
\begin{aligned}
& E\left[\hat{P}^{(\mathrm{u})}((s, t) \in(x, y) \times(x+\Delta x, y+\Delta y))\right] \\
= & \iiint \rho^{(\mathrm{u})}(s, t, r) I((s, t) \in(x, y) \times(x+\Delta x, y+\Delta y)) \mathrm{d} s \mathrm{~d} t \mathrm{~d} r \\
= & \iiint_{(s, t) \in(x, y) \times(x+\Delta x, y+\Delta y), r} \rho^{(\mathrm{u})}(s, t, r) \mathrm{d} s \mathrm{~d} t \mathrm{~d} r \\
= & P^{(\mathrm{u})}((s, t) \in(x, y) \times(x+\Delta x, y+\Delta y))
\end{aligned}
$$

So, the estimated probability is unbiased.

\section{References and Notes}

(1) Schulz, G. E.; Muller, C. W.; Diederichs, K. J. Mol. Biol. 1990, $213,627$.

(2) Sheng, X. R.; Li, X.; Pan, X. M. J. Biol. Chem. 1999, 274, 22238. 
(3) Han, Y.; Li, X.; Pan, X. M. FEBS Lett. 2002, 528, 161.

(4) Zhang, H. J.; Sheng, X. R.; Niu, W. D.; Pan, X. M.; Zhou, J. M. J. Biol. Chem. 1998, 273, 7448.

(5) Burlacu-Miron, S.; Gilles, A. M.; Popescu, A.; Bârzu, O.; Craescu,

C. T. Eur. J. Biochem. 1999, 264, 765 .

(6) Wolf-Watz, M.; Thai, V.; Henzler-Wildman, K.; Hadjipavlou, G.; Eisenmesser, E. Z.; Kern, D. Nat. Struct. Mol. Biol. 2004, 11, 945.

(7) Sinev, M. A.; Sineva, E. V.; Ittah, V.; Haas, E. Biochemistry 1996, 35,6425 .

(8) Byeon, L.; Shi, Z.; Tsai, M. D. Biochemistry 1995, 34, 3172

(9) Koshland, D. E. Proc. Natl. Acad. Sci. U.S.A. 1958, 44, 98.

(10) Gerstein, M.; Schulz, G.; Chothia, C. J. Mol. Biol. 1993, $229,494$.

(11) Kumar, S.; Sham, Y. Y.; Tsai, C.-J.; Nussinov, R. Biophys. J. 2001 $80,2439$.

(12) Hayward, S. J. Mol. Biol. 2004, 339, 1001.

(13) Eisenmesser, E. Z.; Millet, O.; Labeikovsky, W.; Korzhnev, D. M.; Wolf-Watz, M.; Bosco, D. A.; Skalicky, J. J.; Kay, L. E.; Kern, D. Nature 2005, $438,117$.

(14) Taniuchi, H.; Anfinsen, C. B. J. Biol. Chem. 1966, 241, 4366.

(15) Markley, J. L.; Williams, M. N.; Jardetzky, O. Proc. Natl. Acad. Sci. U.S.A. 1970, 65, 645.

(16) Chazin, W. J.; Kordel, J.; Drakenberg, T.; Thulin, E.; Brodin, P.; Grundstrom, T.; Forsen, S. Proc. Natl. Acad. Sci. U.S.A. 1989, 86, 2195.

(17) Wilson, M. A.; Brunger, A. T. J. Mol. Biol. 2000, 301, 1237.

(18) Lou, H. F.; Cukier, R. I. J. Phys. Chem. B 2006, 110, 12796.

(19) Temiz, N. A.; Meirovitch, E.; Bahar, I. Proteins: Struct., Funct., Bioinf. 2004, 57, 468 .

(20) Miyashita, O.; Onuchic, J. N.; Wolynes, P. G. Proc. Natl. Acad. Sci. U.S.A. 2003, 100, 12570

(21) Maragakis, P.; Karplus, M. J. Mol. Biol. 2005, 352, 807.

(22) Berg, B. A.; Neuhaus, T. Phys. Lett. B 1991, 267, 249.

(23) Hansmann, U. H. E.; Okamoto, Y.; Eisenmenger, F. Chem. Phys. Lett. 1996, 259, 321.

(24) Lyubartsev, A. P.; Martsinovski, A. A.; Shevkunov, S. V.; Vorontsov-Velyaminov, P. N. J. Chem. Phys. 1992, 96, 1776.

(25) Lyubartsev, A.; Laaksonen, A. Appl. Parallel Comput. 1998, 1541 , 296.

(26) Swendsen, R. H.; Wang, J. S. Phys. Rev. Lett. 1986, 57, 2607.

(27) Sugita, Y.; Okamoto, Y. Chem. Phys. Lett. 1999, 314, 141

(28) Hansmann, U. H. E. Chem. Phys. Lett. 1997, 281, 140.

(29) Geyer, C. J. Markov Chain Monte Carlo Maximum Likelihood. In Computing Science and Statistics: Proceedings of the 23rd Symposium on the Interface; Keramidas, E. M., Ed.; Interface Foundation: Fairfax Sta tion, VA, 1991.

(30) Wang, J. S.; Swendsen, R. H. Prog. Theor. Phys. Suppl. 2005, 317.

(31) Hukushima, K.; Nemoto, K. J. Phys. Soc. Jpn. 1996, 65, 1604.

(32) Fukunishi, H.; Watanabe, O.; Takada, S. J. Chem. Phys. 2002, 116 , 9058.

(33) Frenkel, D.; Smit, B. Understanding Molecular Simulation: From Algorithms to Applications; Academic: San Diego, 1996.
(34) Predescu, C.; Predescu, M.; Ciobanu, C. V. J. Phys. Chem. B 2005 , 109,4189

(35) Sugita, Y.; Kitao, A.; Okamoto, Y. J. Chem. Phys. 2000, 113, 6042.

(36) van Gunsteren, W. F.; Billeter, S. R.; Eising, A. A.; Hünenberger,

P. H.; Krüger, P.; Mark, A. E.; Scott, W. R. P. Biomolecular Simulation:

The GROMOS96 Manual and User Guide; Vdf Hochschulverlag AG an der ETH: Zürich, 1996.

(37) Essmann, U.; Perera, L.; Berkowitz, M. L.; Darden, T.; Lee, H.; Pedersen, G. L. J. Chem. Phys. 1995, 103, 8577.

(38) Müller, C. W.; Schlauderer, G. J.; Reinstein, J.; Schulz, G. E. Structure 1996, 4, 147.

(39) Cox, T. F.; Cox, M. A. A. Multidimensional Scaling, 2nd ed.; Chapman \& Hall: Boca Raton, FL, 2001.

(40) García, A. E. Phys. Rev. Lett. 1992, 68, 2696.

(41) García, A. E.; Blumenfeld, R.; Hummer, G.; Krumhansl, J. A. Physica D 1997, 107, 225 .

(42) Amadei, A.; Linssen, A. B. M.; Berendsen, H. J. C. Proteins Struct., Funct., Genet. 1993, 17, 412

(43) Romo, T. D.; Clarage, J. B.; Sorensen, D. C.; Phillips, G. N. Proteins: Struct., Funct., Genet. 1995, 22, 311 .

(44) Teodoro, M. L.; Phillips, G. N.; Kavraki, L. E. J. Comput. Biol. 2003, 10, 617.

(45) Amadei, A.; Ceruso, M. A.; Di Nola, A. Proteins: Struct., Funct., Genet. 1999, 36, 419.

(46) Hess, B. Phys. Rev. E 2000, 62, 8438

(47) Hess, B. Phys. Rev. E 2002, 65, 031910.

(48) Lou, H. Analyzer, version 1.0; East Lansing, MI, 2005.

(49) Kofke, D. A. J. Chem. Phys. 2002, 117, 6911.

(50) Chempath, S.; Predescu, C.; Bell, A. T. J. Chem. Phys. 2006, 124, 234101.

(51) Kumar, S.; Bouzida, D.; Swendsen, R. H.; Kollman, P. A.; Rosenberg, J. M. J. Comput. Chem. 1992, 13, 1011.

(52) Souaille, M.; Roux, B. Comput. Phys. Commun. 2001, 135, 40.

(53) Balsera, M. A.; Wriggers, W.; Oono, Y.; Schulten, K. J. Phys. Chem. 1996, 100, 2567.

(54) McCammon, A.; Harvey, S. C. Dynamics of Proteins and Nucleic Acids; Cambridge University Press: Cambridge, 1987.

(55) Zhang, W.; Wu, C.; Duan, Y. J. Chem. Phys. 2005, 123.

(56) Sanbonmatsu, K. Y.; Garcia, A. E. Proteins: Struct., Funct., Genet. 2002, 46, 225.

(57) Silverman, B. W. Density Estimation for Statistics and Data Analysis; Chapman and Hall: London, New York, 1986.

(58) Müller, C. W.; Schulz, G. E. J. Mol. Biol. 1992, 224, 159.

(59) Walsh, C. Enzymatic Reaction Mechanisms; W. H. Freeman: San Francisco, 1979.

(60) Hayward, S.; Lee, R. A. J. Mol. Graphics Modell. 2002, 21, 181. 2132

(61) Bae, E.; Phillips, G. N. Proc. Natl. Acad. Sci. U.S.A. 2006, 103, 